\title{
Pose Measurement Performance of the Argon Relative Navigation Sensor Suite in Simulated Flight Conditions
}

\author{
Joseph M. Galante, John Van Eepoel’ Matt Strube $\ddagger$ Nat Gill ${ }^{\ddagger}$ \\ NASA Goddard Spaceflight Center, Greenbelt, MD, 20771, USA \\ Marcelo Gonzalez, Dr. Andrew Hyslop§, Bryan Patrick \\ Emergent Space Technologies, Greenbelt, MD, 20770, USA
}

\begin{abstract}
Argon is a flight-ready sensor suite with two visual cameras, a flash LIDAR, an onboard flight computer, and associated electronics. Argon was designed to provide sensing capabilities for relative navigation during proximity, rendezvous, and docking operations between spacecraft. A rigorous ground test campaign assessed the performance capability of the Argon navigation suite to measure the relative pose of high-fidelity satellite mockups during a variety of simulated rendezvous and proximity maneuvers facilitated by robot manipulators in a variety of lighting conditions representative of the orbital environment. A brief description of the Argon suite and test setup are given as well as an analysis of the performance of the system in simulated proximity and rendezvous operations.
\end{abstract}

\section{Nomenclature}

$\begin{array}{ll}\text { Pose } & \text { Position and orientation of an object relative to another } \\ \text { Point cloud } & \text { Collection of points given in a 3D coordinate system } \\ \bar{a} & \text { Measurement of vector } a \\ \hat{a} & \text { Estimate of vector } a \\ { }^{C} a & \text { Vector } a, \text { measured in coordinate frame } C \\ c m & \text { Center of mass } \\ r & \text { Position vector } \\ q & \text { Attitude quaternion } \\ v & \text { Velocity vector } \\ \omega & \text { Attitude rate vector } \\ F & \text { Force vector } \\ T & \text { Torque vector }\end{array}$

\section{Introduction}

In-space rendezvous is a key enabling technology for many of NASA's current and planned space missions, especially in the areas of human exploration, multiplatform scientific investigations, and in-space servicing. ${ }^{1}$ The Satellite Servicing Capabilities Office (SSCO) at the Goddard Space Flight Center (GSFC) is actively working to mature relative navigation sensors and algorithms necessary to rendezvous with a client spacecraft in geosynchronous orbit in order to provide refuel, repair, and reposition services. However, unlike most rendezvous missions, these client satellites are assumed to be non-cooperative: they have no cooperative retroreflectors or sensors, they do not have docking or grapple features, and some form of robotic manipulator

\footnotetext{
*Aerospace Engineer, Attitude Control Systems Engineering Branch, NASA Goddard Space Flight Center, and AIAA Student Member

${ }^{\dagger}$ Aerospace Engineer, Attitude Control Systems Engineering Branch, NASA Goddard Space Flight Center, and AIAA Professional Member

$¥$ Aerospace Engineer, Component Hardware and Systems Branch, NASA Goddard Space Flight Center

$\S$ AIAA Professional Member
} 
is required to capture a suitable feature such as a satellite's Marman ring, the location where the satellite structure connects with the launch vehicle. Moreover, these applications require a high level of autonomy during the critical steps prior to capture of the client spacecraft.

To address these unique technical challenges, the SSCO has developed the Argon system: sensors, algorithms, processors, and ground terminal integrated in a flight-like configuration. This system was used in a series of ground-based rendezvous and proximity operations tests to autonomously determine the relative position and attitude of simulated targets over separation distances ranging from two to 90 meters. Tests were conducted over a period of eight months in 2011 and 2012 using facilities at GSFC and the Naval Research Laboratory (NRL). This paper will describe the Argon system and the test campaign, as well as present selected performance results for the sensors and estimation algorithms tested.

\section{Argon Relative Navigation System}

The Argon system comprises all the major elements - sensors, processing hardware, and algorithmsnecessary for a Rendezvous and Proximity Operations (RPO) subsystem within a servicing spacecraft. The Argon system leveraged hardware and software developed as part of the Dextre Pointing Package (DPP) development program, a proposed relative navigation sensor suite to be installed as an external payload on the International Space Station (ISS). ${ }^{2}$ Figure 1 provides a translucent model showing Argon's internal components and a photograph taken during system integration.
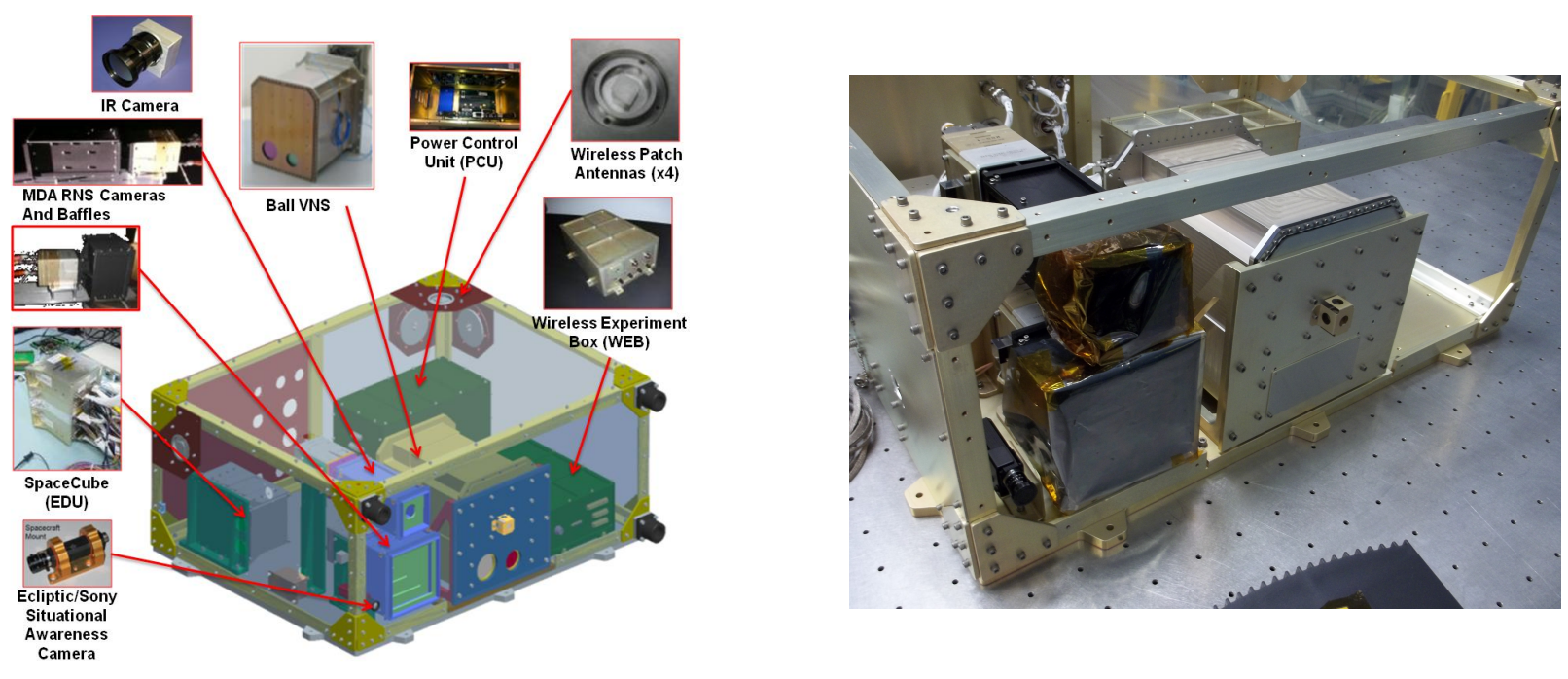

Figure 1. Argon features a number of relative navigation sensors in addition to the necessary power, communications, and processing electronics in a flight-like package (left). Argon system during integration; cameras and flash LIDAR are visible (right).

\section{A. Sensing Hardware}

Two one-megapixel optical cameras, built by MacDonald, Dettwiler and Associates (MDA), provide monochrome visible-wavelength images for processing by the optical pose algorithm. The two cameras have different fields of view, 11 and 57 degrees, that are optimized for a particular range to the target. The camera units included in Argon were the flight cameras used during the Relative Navigation Sensor (RNS) experiment that flew on STS-125 as part of the Hubble Space Telescope (HST) Servicing Mission 4 (SM4). ${ }^{3}$

Also included in Argon is a Vision Navigation System (VNS) flash Light Detection and Ranging (LIDAR), designed and built by Ball Aerospace technologies Corporation. VNS is the primary relative navigation sensor for the Orion Multi-Purpose Crew Vehicle. The VNS unit used in Argon was assembled using surplus hardware developed under the Orion Project, and in partnership with the NASA Engineering and Safety Center (NESC) Autonomous Rendezvous and Docking (AR\&D) Community of Practice. The VNS uses a near-infrared laser with a 20 degree beam divergence to illuminate the scene while a 256 x 256 PIN diode array measures both return intensity and range on a per pixel basis. The resulting data are in the form of a point 
cloud, a set of 3D points referenced to a coordinate frame fixed to the LIDAR. An earlier version of the VNS sensor flew as a cooperative sensor as part of the Sensor Test for Orion Rel-Nav Risk Mitigation (STORRM) Demonstration Test Objective (DTO) on the STS-134 mission. ${ }^{4}$

\section{B. Pose Software}

Argon hosts the Goddard Natural Feature Image Recognition (GNFIR) algorithm to provide pose measurements from the 2D optical camera images. GNFIR extracts recognizable features from the image data and then matches them to a model of the target (formulated by a set of edges). Based on constrained contour tracking, ${ }^{5}$ GNFIR is a complete pose package that includes a general acquisition strategy for generic targets, range-assisted acquisition, firmware-accelerated edge detection and tracking, and active feedback to a custom camera automatic gain control (AGC) algorithm. A previous version of this algorithm also successfully flew during the RNS experiment on STS- $125 .^{3}$

Argon also hosts the FlashPose (FPose) algorithm, which processes real-time flash LIDAR frames to produce a six degree of freedom (DOF) pose estimate. The algorithm processes range images as a point cloud, samples the cloud using tunable quality metrics, and uses a custom implementation of the Iterative Closest Point (ICP) algorithm ${ }^{6}$ to determine an optimal estimate of the relative position and attitude. FPose also provides an independent 3DOF relative position measurement: this is useful when the number of valid returns in the point cloud shrinks as the target moves farther away. Similar to GNFIR, the FPose algorithm can be run on the Argon system or via the ground system using the Vispose application. Figure 2 shows screen shots from Vispose, a ground application used to graphically display algorithm telemetry, of GNFIR and FPose tracking a satellite model during the Argon test campaign.
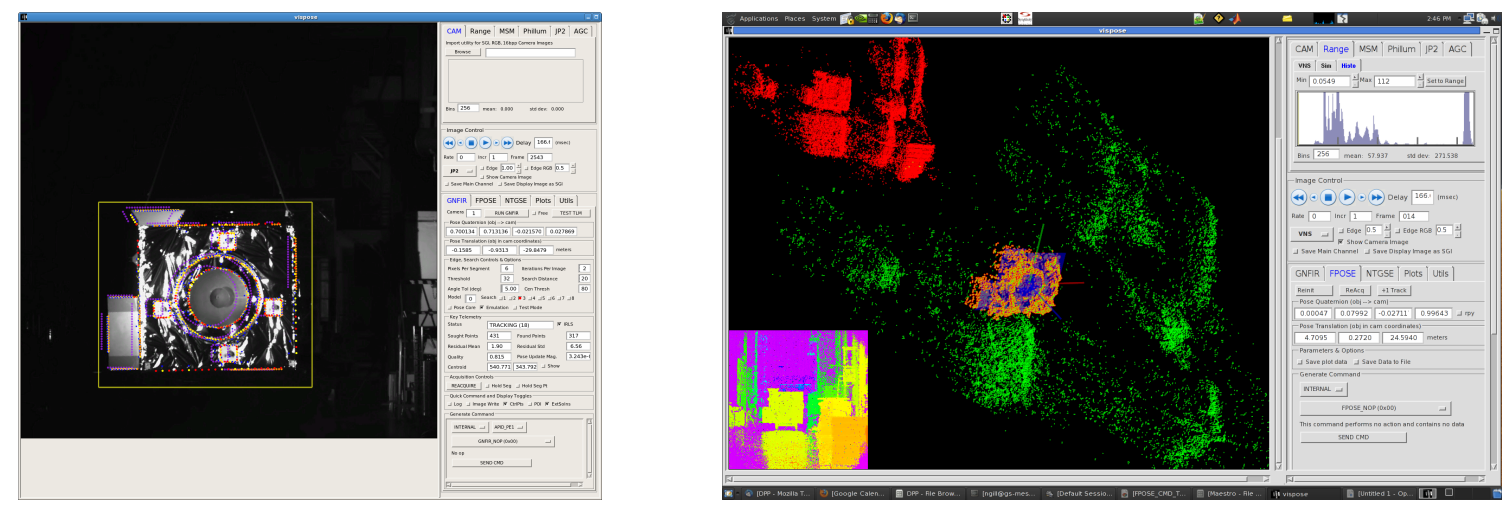

Figure 2. Screen shots from the Vispose Graphical User Interface running Argon non-cooperative pose algorithms, GNFIR (left), FPose (right). The GNFIR instance of Vispose shows a raw camera feed, the subsampled window outline depicted at a box, and the GNFIR pose estimate. The FPose instance of Vispose contains a raw range LIDAR feed in the bottom left, a model in the center, and several point clouds generated from the LIDAR data.

\section{Processing Hardware}

The core processing unit in Argon is the GSFC-developed SpaceCube, ${ }^{7}$ a hybrid computing platform designed to provide command and data handling functions for earth-orbiting satellites. The SpaceCube is comprised of five slices (cards): two Power Slices, two Processor Slices, and one Video Control Module (VCM) Slice. Each processor slice contains two Xilinx Virtex 4 Field Programmable Gate Arrays (FPGAs) and each FPGA contains two PPC405 processors running at $250 \mathrm{MHz}$. These eight processors host multiple instantiations of the Argon pose applications GNFIR and FPose, along with command and telemetry handling software that allows a flight-like ground terminal to control Argon remotely. The VCM provides sensor data compression and $16 \mathrm{~Gb}$ of flash memory to store raw sensor images for later playback. The Argon SpaceCube is an engineering development unit (EDU) version of the hardware flown on the RNS experiment and Materials International Space Station Experiment 7 (MISSE-7). 


\section{Visual Targets}

The GOES-12 spacecraft, a geostationary weather satellite operated by the National Oceanic and Atmospheric Administration (NOAA), was selected as the example client satellite for Argon testing. GOES-12 is a government-owned satellite that is a possible candidate for a robotic refueling demonstration and is typical of other candidate servicing clients in that it was not designed with any features to facilitate rendezvous and docking.

Two high-fidelity models of the GOES-12 spacecraft were developed for Argon testing: a full-scale mockup of the west deck and a tenth-scale model of the entire satellite. The full-scale mockup is used when the servicing vehicle is assumed to be aligned with the client and slowly approaching in preparation for final capture of the Marman ring, which is located on the west deck. The model was constructed from engineering drawings and close-out photos of the actual GOES-12 satellite. Flight-like materials and blankets were used to create realistic features similar to what might be seen on orbit. Additional features were added to the model before the final series of tests conducted at the NRL. Figure 3 shows the full-scale mockup before and after upgrades. Tests were conducted with the full-scale mockup for separation distances ranging from 2 to 90 meters.

The tenth-scale model, shown in Figure 4, allows for a wider range of relative motion than just along the final approach direction. The scaling of the model allows sensor-client separations to be simulated on a 10:1 ratio, making it easier to simulate distances greater than 100 meters in a controlled lab environment. The completeness of the model allows for testing complex shadowing conditions, fly-arounds, and inspection operations. The project also built a variety of other shape and material targets that were used early in Argon testing to help characterize the individual sensors.
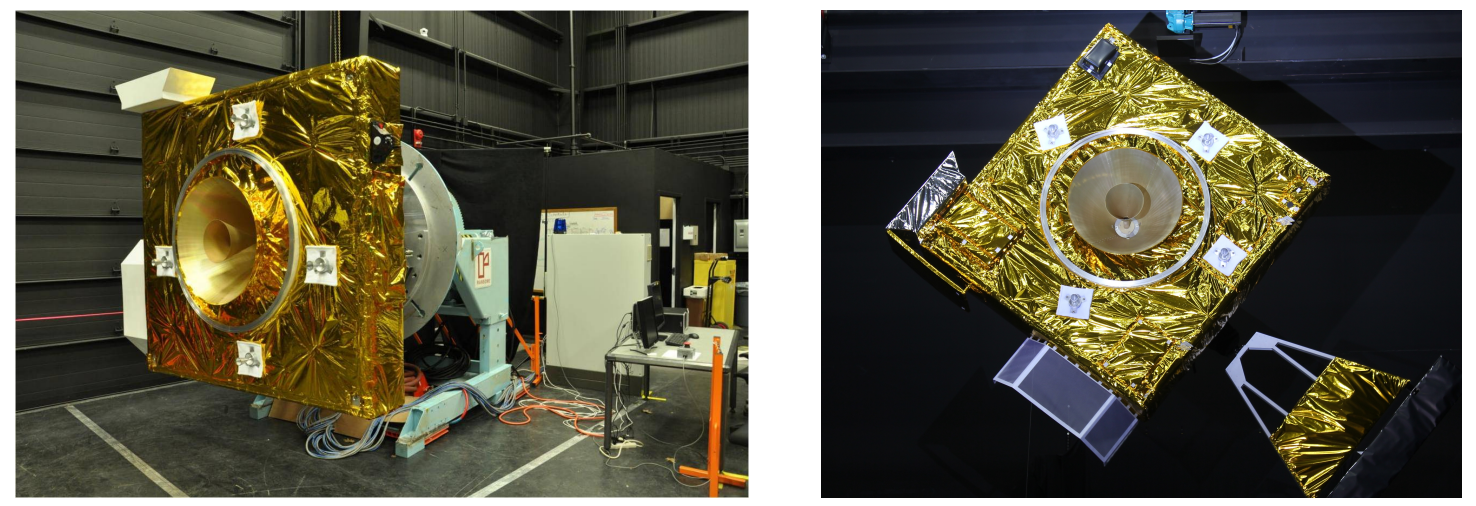

Figure 3. Pictures of full-scale mockup as used in the SSCO (left) and NRL (right) test facilities. Upgrades to the model were made based on improved GOES-12 close-out pictures, including a partial solar array, prior to final series of NRL tests. (Credit: NASA (left), NRL (right))

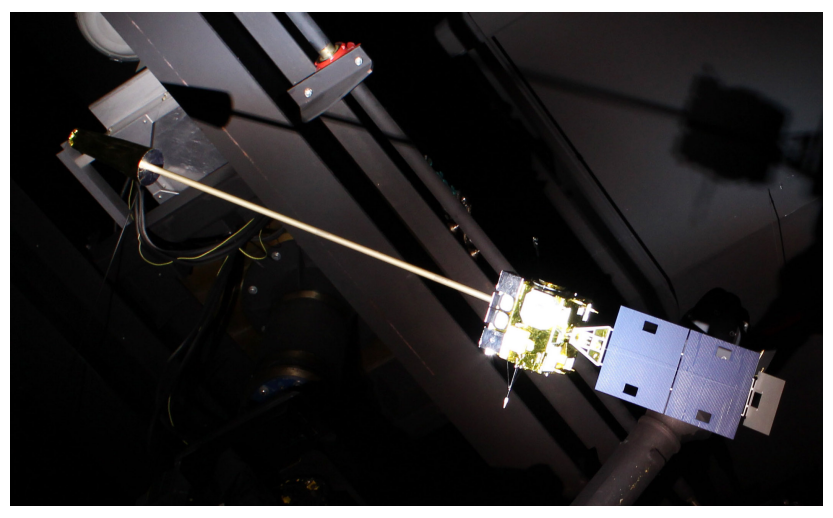

Figure 4. Picture of tenth-scale model of GOES-12 during testing at NRL. (Credit: NRL) 


\section{E. Test Operations}

In part because it was originally developed as the DPP ISS payload, Argon operates in a manner very similar to how the actual flight system would. A comprehensive ground terminal has been developed and implemented via GSFC standard Advanced Spacecraft Integration \& System Test Software (ASIST) workstations $^{8}$ to send commands to and receive telemetry from Argon. During test operations Argon's sensors record images of the spacecraft target as it moves through some pre-determined motion. Real-time sensor data is processed with GNFIR and FPose applications onboard the Argon SpaceCube; compressed images and onboard pose solutions are telemetered to the ground system in real-time. Ground-based workstations run independent GNFIR and FPose algorithms to monitor onboard algorithm performance (see Figure 2). Commands are issued from the ground system to configure sensor settings, change AGC settings, reconfigure telemetry, or re-initialize the onboard algorithms.

\section{Argon Test Campaign}

Argon system testing was conducted using facilities at both GSFC and NRL to simulate varying distances, relative motion, and realistic environmental conditions. First, at the GSFC Integration and Test Complex, GNFIR and FPose were demonstrated in the end-to-end system, as well as evaluated as a function of range. Second, the algorithms were tested for relative translation and rotation rate robustness at the GSFC Satellite Servicing Center. Finally, the complete system was integrated in closed-loop tests conducted at the NRL Proximity Operations Testbed (POT) facility where large-scale 6DOF relative motion capabilities allowed testing of a final approach scenario. Each test location and objective is covered in more detail in the following sections.

\section{A. Relative Range Performance Characterization}

This section describes the first phase of Argon system testing completed at the GSFC Integration and Testing Complex from November to December 2011. While the primary objective was to demonstrate an end-to-end functional system, the layout of the Integration and Testing Complex also allowed for the characterization of the accuracy of both pose algorithms as a function of range. This section contains data from an example run for each of the two pose algorithms as well as a condensed plot of the observed performance as a function of range.

\section{Experimental Setup}

The tests described in this section were conducted with the Argon hardware located in a positive pressure clean tent. The positive pressure clean tent is where the system was integrated and component-level tested, so this location allowed for hardware configuration updates as we broke in the system. The client targets were moved along a 5-meter-wide, 90-meter-long hallway that was co-linear with the opening in the clean tent: during tests, the side of the clean tent would be rolled up to give the sensors an unobstructed view of the target. The client targets were moved dynamically relative to the sensors using a wheeled cart or an overhead crane, while the sensors were manually pointed at the targets using a custom-built turn-over dolly. The image on the left of Figure 5 shows the Argon system on the dolly and the full-scale mockup installed on a wheeled cart. The center picture shows the clean tent and its location with respect to the 90 meter hallway. The picture on the right of Figure 5 shows the full-scale mockup suspended on the crane during one of the tests. Beyond changing the separation distance, the cart and crane also allowed relative dynamic motions to be introduced in three and four degrees of freedom, respectively, to stress the system.

To gauge performance, the reported pose solutions from Argon were compared to truth metrology taken from a Leica AT901-MR laser tracking system equipped with the T-Mac and T-Cam accessories. This metrology system is capable of measuring the position and orientation of a dynamic target, with respect to any defined working frame, at $10 \mathrm{~Hz}$ with an accuracy of $\pm 70 \mu \mathrm{m}$ in position and $0.01^{\circ}$ in attitude, which is well below the accuracy of the system under test.

The test plan consisted of over 100 runs which varied four parameters: separation distance, lighting configurations, relative orientation (in one axis), and dynamic motions. The main variable of interest was separation distance, which was changed in 5-meter increments from 5 to 65 meters. The angle of incidence of the light source was varied between 15, 45, and 90 degrees. Relative attitude was varied in yaw (out- 

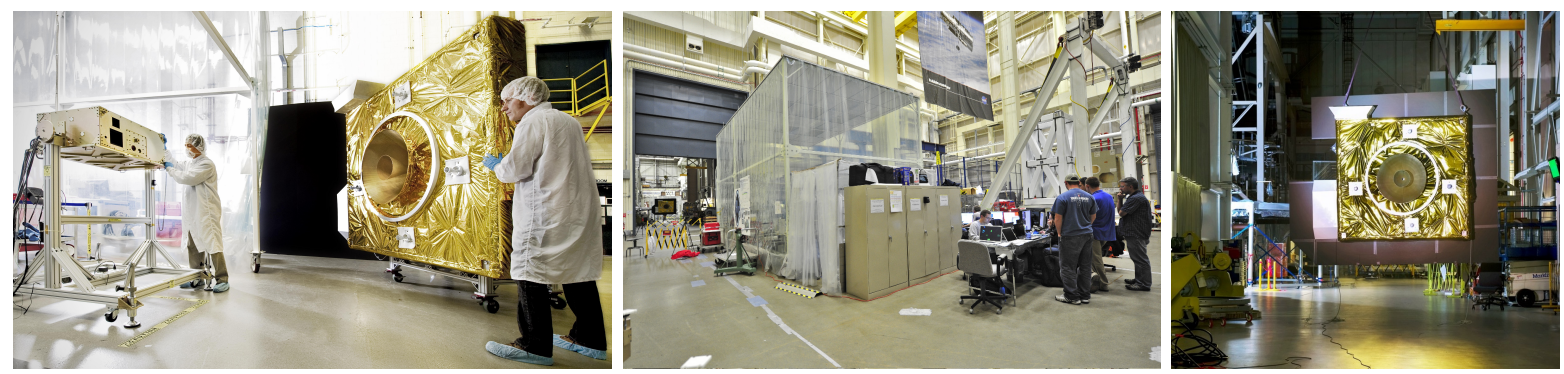

Figure 5. Pictures showing the concept of operations for the Spot 5 testing. From left: Argon and the Full Scale GOES-12 model; Argon operations in the foreground with target suspended in the background; close up of target illuminated by spotlight during testing. (Credit: NASA/Chris Gunn)

of-plane rotations) which were keep at 0 degrees or varied dynamically during a test between \pm 30 degrees; pitch and roll were not varied at this location as neither the cart nor the crane allowed for changes in those axes. Finally, separation distance, side-to-side, and up-and-down motions were all varied by the actuating the overhead crane. The range of the in-plane dynamic motions (up/down and left/right motions) were 1-2 meters. The separation distance was varied between 5-10 meters. While the test configuration at this location was capable of introducing 60 meters of separation distance changes - using the overhead crane - the Leica system had a range limitation that reduced the dynamic motions to the quoted levels. The velocities for translational dynamic motions were limited to approximately $2 \mathrm{~cm} / \mathrm{sec}$ when using the crane. While real-time telemetry from GNFIR and FPose were obtained during the tests, the error analysis that follows uses solutions generated at a later date using recorded images from actual test runs.

\section{Results}

Figures 6 and 7 contain sample output of GNFIR and FPose, respectively, during a single data run. The plots along the top row in each figure show the reported relative position (left) and attitude (right), while the plots along the bottom row show the RSS in-plane and out-of-plane position and attitude errors with respect to the Leica solution. The GNFIR data are from a test where the full-scale mockup hanging from the crane and moving from 55 to 45 meters away. The FPose data show a test where the target exhibited dynamic, in-plane motion at a separation of 65 meters.

For each tested separation distance, 100 individual pose solutions from both algorithms were used to generate a Root Mean Square (RMS) value and standard deviation of the in-plane and out-of-plane errors. The RMS and standard deviation values were then plotted as a function range which is shown in Figure 8 and 9 for GNFIR and FPose, respectively. In each figure, the data points represent the RMS error and the hashed boxes represent the associated standard deviations. The GNFIR data are also separated according to favorable and unfavorable lighting, examples of which can be found in Figure 10.

\section{GNFIR Performance}

GNFIR performs similarly over the separation distances tested. One may expect to see accuracy decrease as the separation distance increases; however, for this camera configuration and range of separation distances, the target features used in GNFIR adequately span the camera image, providing favorable geometry. The effect of increased error for increased separation distances can been seen in data presented in Section C.

Also observe that the presence of unfavorable lighting appears to effect performance in determining separation range and pitch and yaw angles. Before testing, unfavorable lighting was assumed to decrease performance uniformly across all axes; however, it is not surprising that separation range and out-of-plane attitudes would be more affected by adverse lighting since these are degrees of freedom that are less observable in a $2 \mathrm{D}$ imaging system.

GNFIR also exhibits pose-dependent measurement biases in all degrees of freedom, which are seen at ranges less than 30 meters where the RMS errors are significantly larger than their associated standard deviations. Pose-dependent biases are produced when the GNFIR algorithm converges on a solution that is an incorrect local minimum. Since the data points used in this analysis are from measuring a target with a constant relative position and attitude, there is no "excitation" to perturb the GNFIR solution out of the 

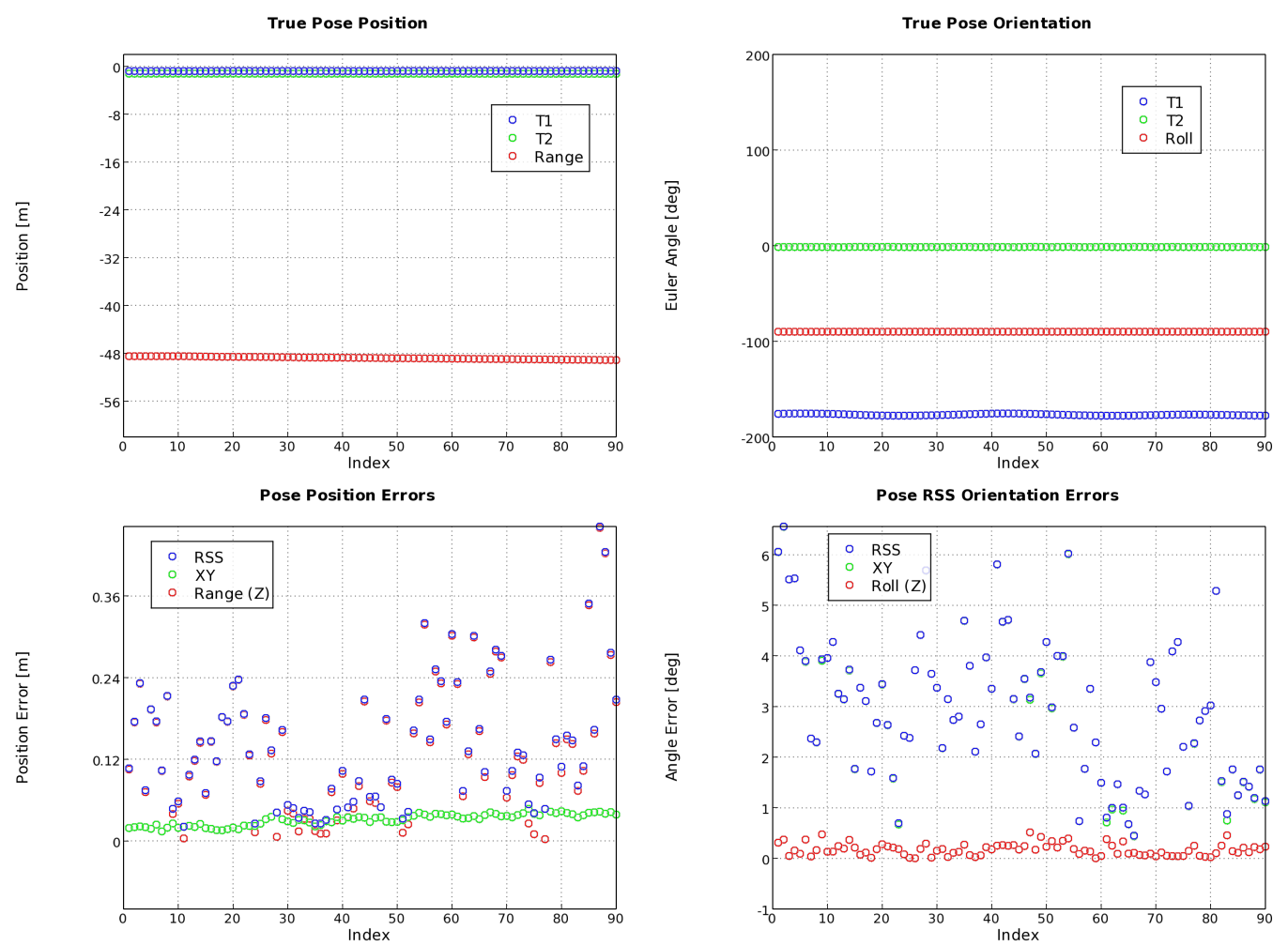

Figure 6. Sample GNFIR solution showing measured pose (top) and error (bottom) over time.
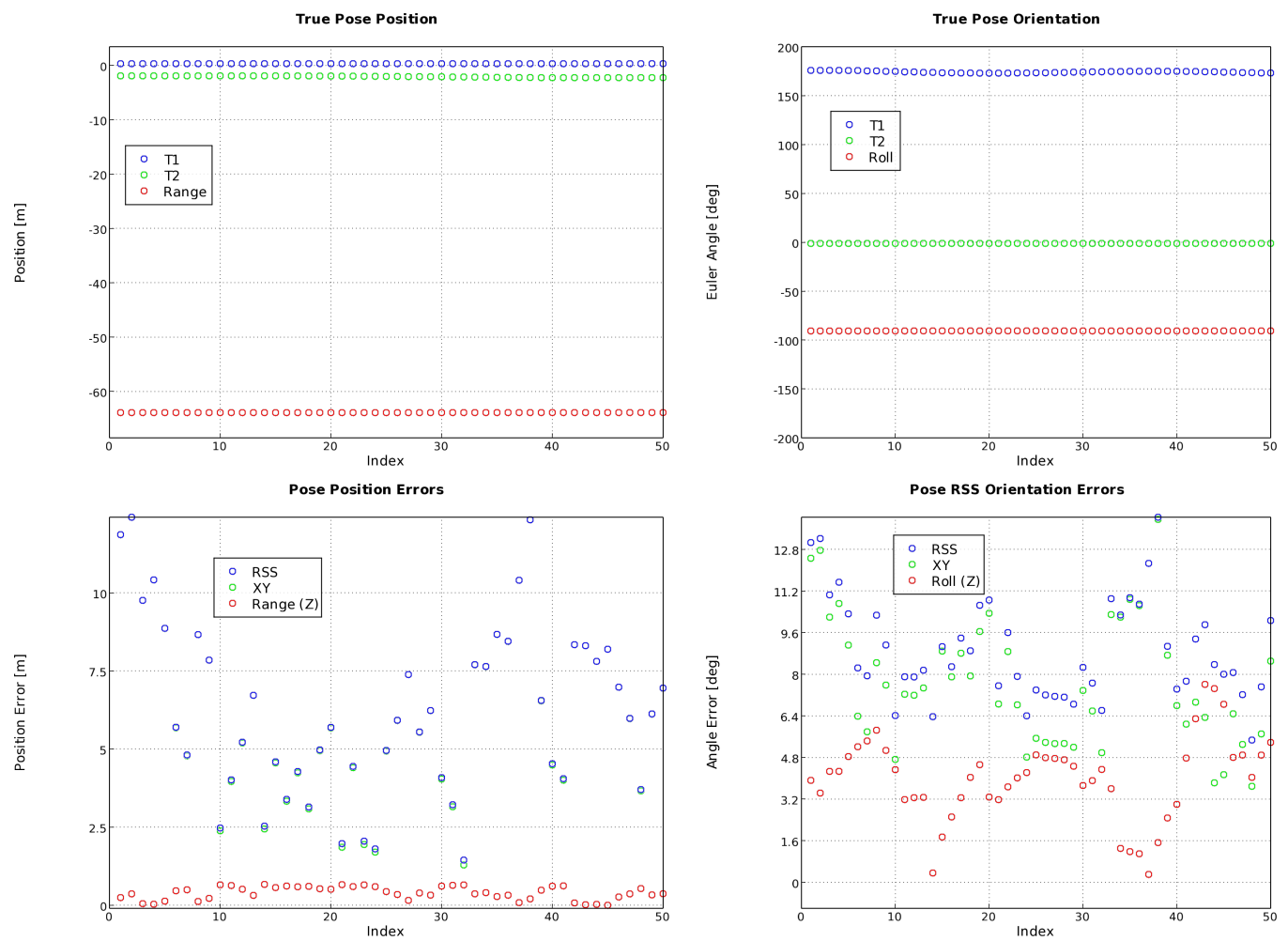

Figure 7. Sample FPose solution showing measured pose (top) and error (bottom) over time. 

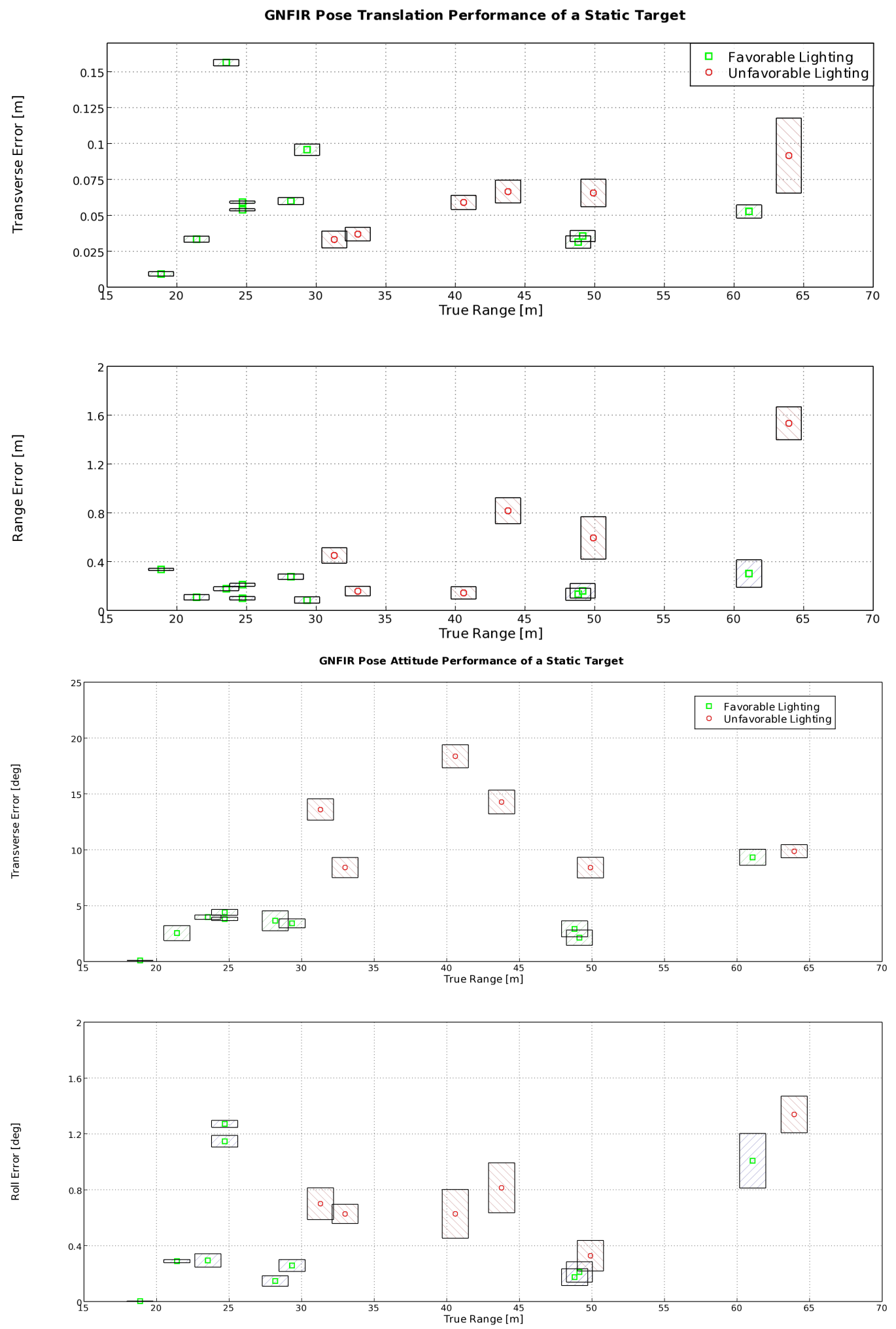

Figure 8. Overall GNFIR errors as a function of range and lighting. Data from 11 degree FOV camera. 

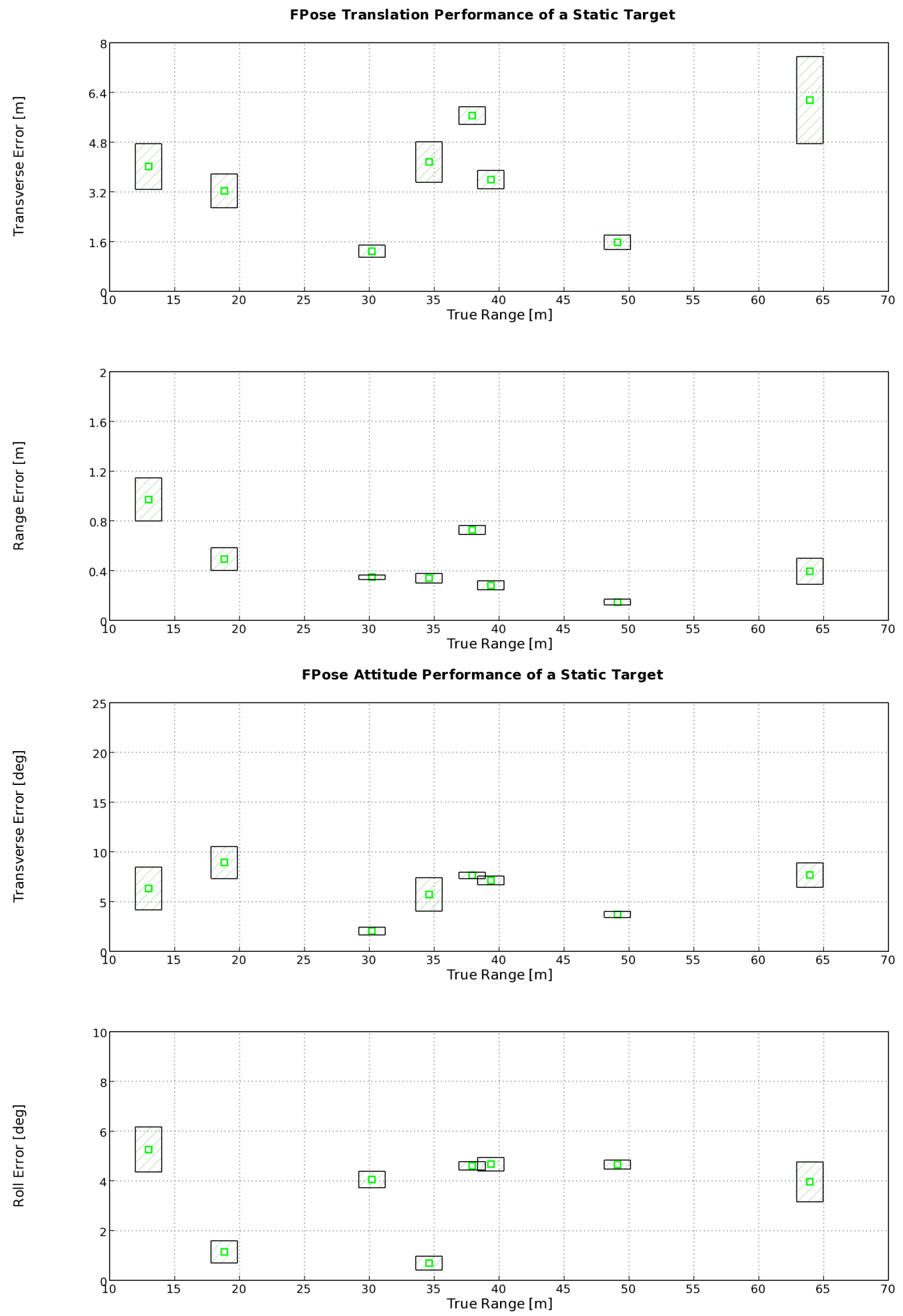

Figure 9. Overall FPose errors as a function of range. 

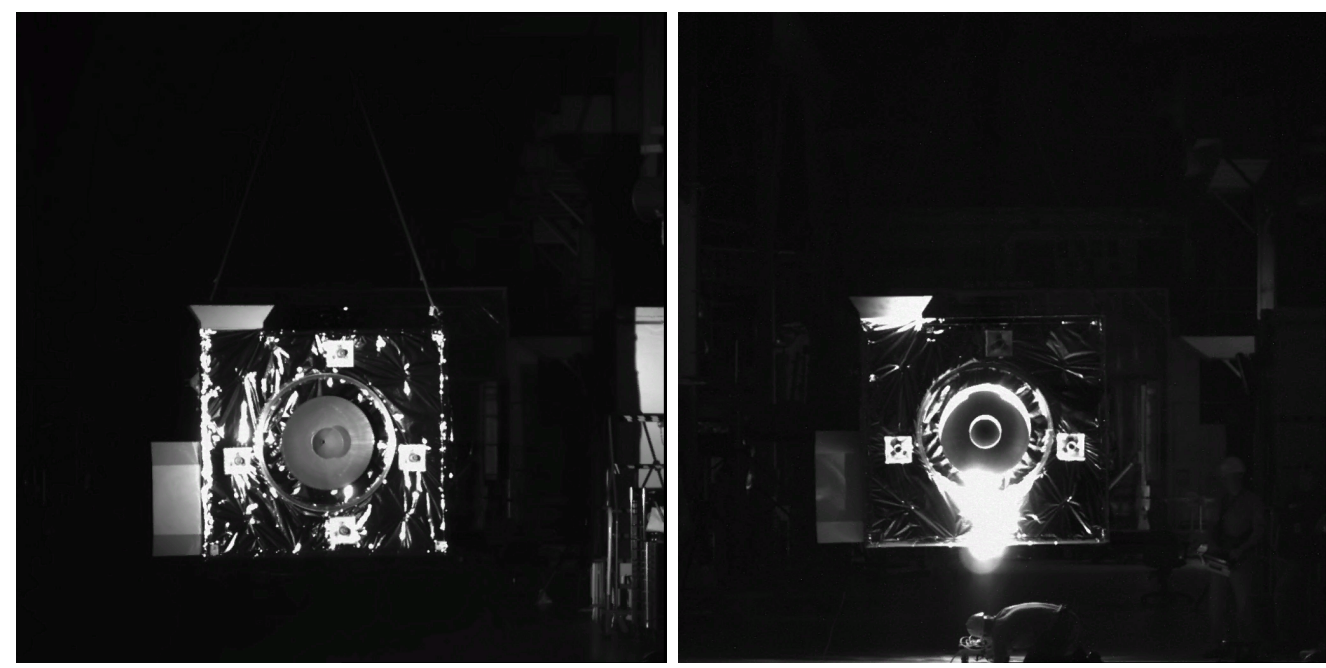

Figure 10. Example lighting conditions of full-scale mockup in GSFC facility: favorable lighting on the left and unfavorable on the right. (Credit: NASA)

local minimum. The results here should be compared with the results from the dynamic tests presented in Section B; when presented with a dynamic target, the pose-dependent biases average out over time, providing a better average solution.

Other sources of error exist for GNFIR that are not range-dependent. For example, the GNFIR algorithm operates by matching features of a model to features in an image. Inaccuracies in the model can erroneously throw off GNFIR's pose estimate. An example of this effect is shown in Figure 11; here, the thruster boxes in the internal model do not match the location of the physical thruster boxes on the target. These modeling errors have the effect of a slight bias in GNFIR's reported position as can be seen in the offset of the Marman ring.

Even if the model is correct, GNFIR is dependent on the quality its raw camera data. One source of bad imagery is incorrect gain and integration interval settings provided to the cameras by the AGC algorithm. The automatic gain control (AGC) algorithm varies the range of light intensities that the camera can record; increasing gain allows dark scenes to be properly exposed but bright scenes get washed out, while decreasing gain allows brighter scenes to be properly exposed but dark scenes appear blacked out. The AGC algorithm tries to maximize information content in a windowed portion of the image, but as seen in the right inset of Figure 11 a number of useful features are overexposed as the gain is too high. The overexposure has the effect of blurring important features that GNFIR is trying to match (in this case, the Marman ring and thruster boxes) which degrades pose estimation performance. Poor gain and integration interval settings from the automatic gain control (AGC) can blur, wash out, or black out important features that GNFIR needs to see, resulting in degraded pose estimation performance.

\section{FPose Performance}

As shown in Figure 9, the performance of FPose using the VNS data was considerably worse than that of GNFIR. In the process of investigating this issue, it was observed that the VNS imagery exhibited rangedependent, non-zero-mean biases for each individual pixel that were on the order of two meters, peak-to-peak. This observation was further confirmed by evaluating VNS imagery of a flat-field target, a 2-meter-square flat plate, at different separation distances, and noticing the deviation between the true ranges on the target and the reported ranges in the data.

Hypothesizing that the VNS data statistics were the source of the FPose algorithm performance anomalies, we attempted to mimic the VNS data using our synthetic imagery generation tool. The tool takes as input a 3D model of the target and a scene definition that provides information about where objects and the LIDAR unit are located in a 3D scene. This scene is then sampled with a stochastic ray-tracing tool to produce ideal point-cloud data. To mimic a specific LIDAR unit, the user is able to adjust a variety of parameters including minimum and maximum range gates, sample rates, detector noise, radiance threshold- 


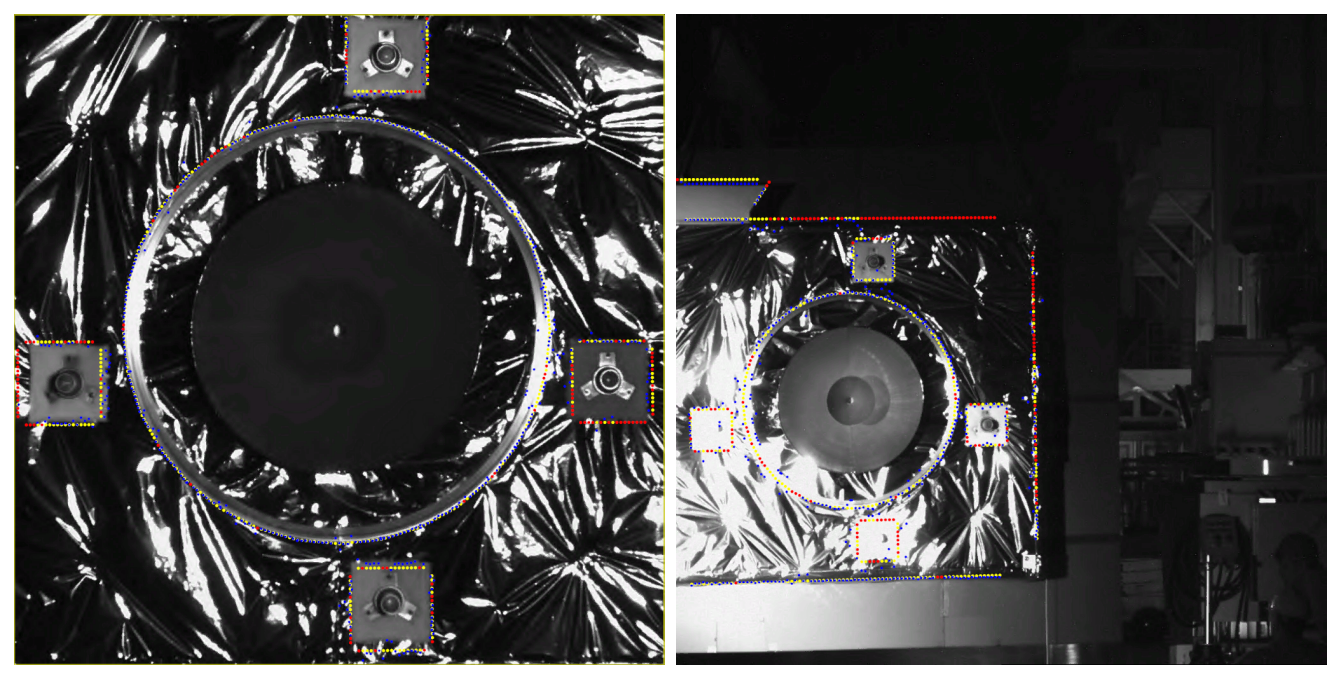

Figure 11. An example of GNFIR overlaying a model fit to features in imagery of a target. Red dots indicate model points that GNFIR was unable to correlate to image features, yellow dots indicate model points that GNFIR was able to correlate with an image feature, and blue dots are the image features that GNFIR matched with a model point. The left inset shows how incorrect placement of the thruster boxes in the model can adversely impact the model fit to the image; the symmetric inaccuracies in the vertical direction "cancel each other out" while the horizontal model errors have the effect of biasing the pose estimate in the transverse direction. The right inset illustrates washed out features due to poor lighting and incorrect AGC settings.

ing, and pulse width. The resulting ray-traced image was then corrupted with the observed VNS statical parameters to produce a simulated VNS image. Figure 12 shows an example VNS image to be simulated, as well as the images produced along the way by the generation process.
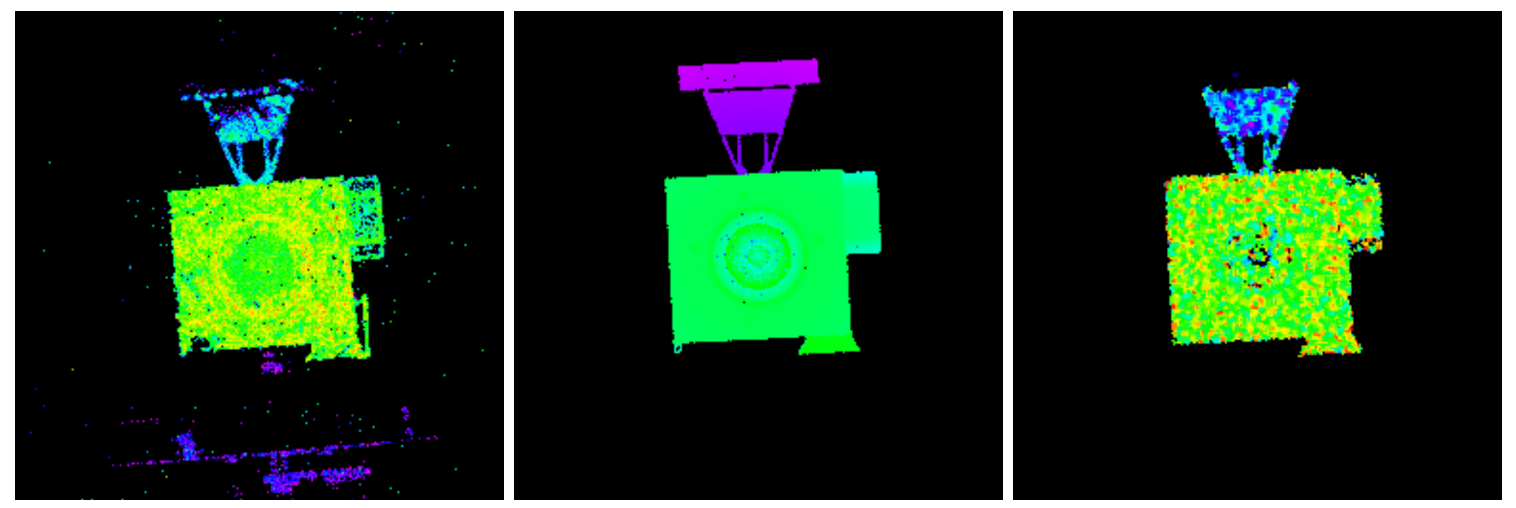

Figure 12. The synthetic imagery generation process. The actual VNS image (left) provides the data for the ray-traced ideal image (center) and the corrupted final image (right).

Using the output from the synthetic imagery generation tool, FPose solution errors were modeled as a function of peak-to-peak pixel biases; the data from this modeling is shown in Figure 13 where the vertical axis displays errors in meters or degrees for position and angular errors, respectively. The errors are consistent with the tests conducted in the Integration and Testing Facility, which lends weight to our hypothesis that the VNS image biases were a significant contributor to the poor FPose performance. Because of the poor performance, FPose was omitted from testing at the other locations.

It should be noted that the VNS performs well when used in its as-designed, cooperative capacity. As used in the Orion Multi-Purpose Crew Vehicle (MPCV) relative navigation sensor suite, the VNS will observe ISS retroreflectors that provide a high signal-to-noise ratio spot in the VNS data. The observed retroreflector locations are then compared to an onboard catalog to generate a 6 -DOF pose measurement. ${ }^{9}$ The VNS manufacturer optimized the VNS design to work in this cooperative environment which is significantly 


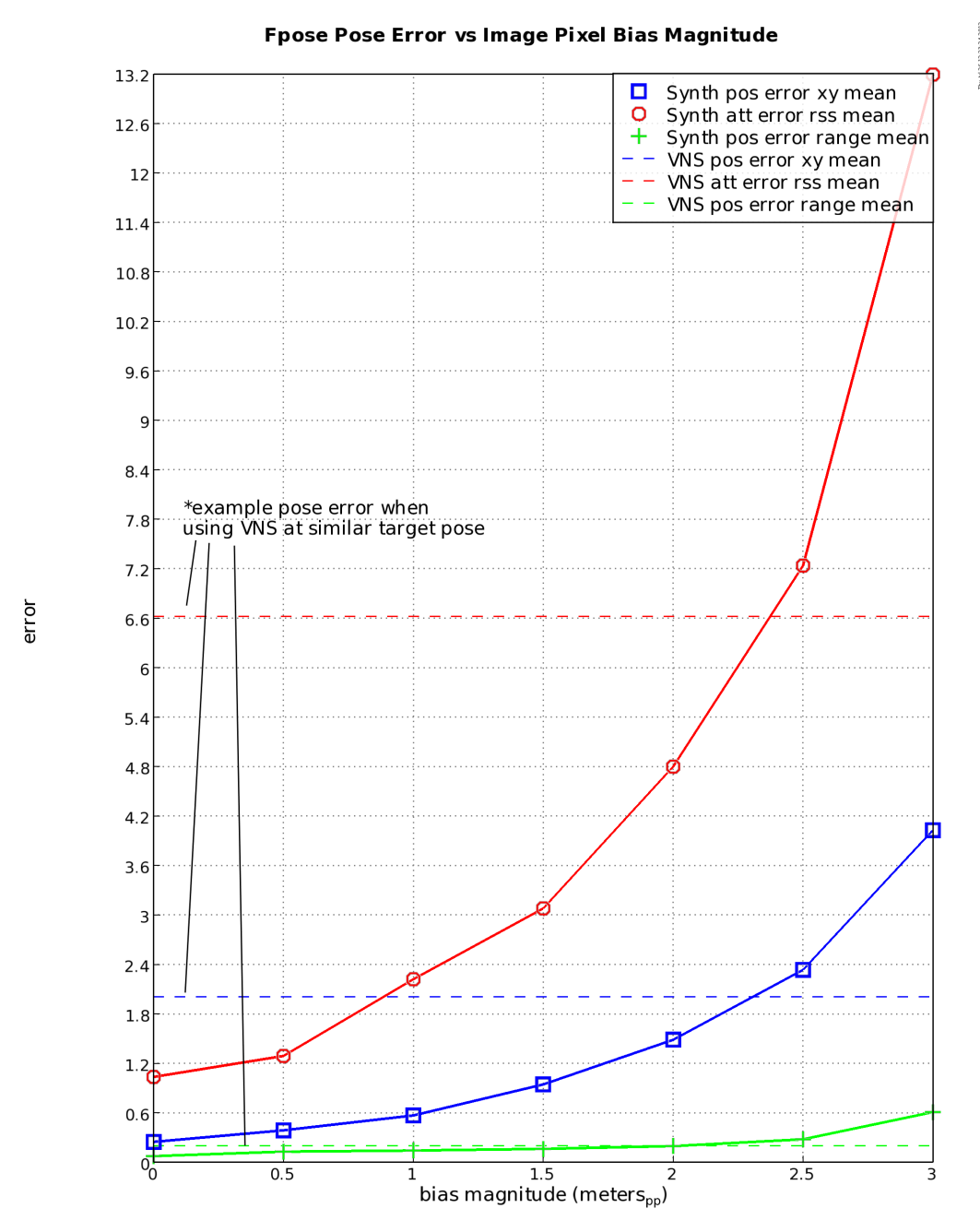

Figure 13. FPose error as a function of peak-to-peak bias errors in synthetic imagery. Horizontal lines shows FPose performance using actual VNS image with same pose.

different than the non-cooperative, feature-tracking environment. One benefit of the synthetic imagery tool described above is that, for specified FPose performance goal, it can generate hard requirements on image data statistics that can be easily tested against for any future LIDAR provider.

\section{B. Target Rate Performance Characterization}

This section describes the second phase of Argon testing that occurred in the GSFC Satellite Servicing Center during January and February of 2012. The Satellite Servicing Center is a unique facility that is used to develop, test, and verify robotic operations intended for the space environment. Most recently this facility has supported tests of robotic tool removal, manipulation, reach, access, and stowage activities in support of on-orbit robotic operations of the ISS for the Robotic Refueling Mission (RRM) mission. ${ }^{10}$ For Argon testing, the Center's multiple robotic manipulation platforms were used to introduce fine relative positioning. This capability allowed the team to bound pose performance as a function of relative velocity and angular rates. The facility also provided the capability to perform initial closed-loop functional testing in advance of the next phase of testing.

\section{Experimental Setup}

The Argon tests conducted in the Satellite Servicing Center used two motion platforms to provide finely controlled relative motion between the Argon and full-scale mockup. The Argon hardware was mounted to 
the end effector of a FANUC 430 robotic manipulator and the target was installed on a Rotopod R-2000 robotic platform, as shown in Figure 14. While only one of these platforms is needed to effect relative motion, two robots provide an increased workspace. The FANUC manipulator was used to provide all of the of the relative translation motion, within a $1 \mathrm{~m}^{3}$ volume, while the Rotopod provided relative attitude motion (up to \pm 15 degrees in pitch and yaw and \pm 720 degrees in roll). To test at different ranges outside the 1-meter volume, the Rotopod was installed on a turn-over dolly sitting on airpads to allow the whole structure to be configured at different initial separation distances. The setup described was tested at ranges of 2,5 , and 10 meters and evaluated against the same Leica truth metrology system used in tests at the Integration and Testing Complex. A more thorough description of this setup and its capabilities as a relative satellite motion simulator can be found in Ref. 11.

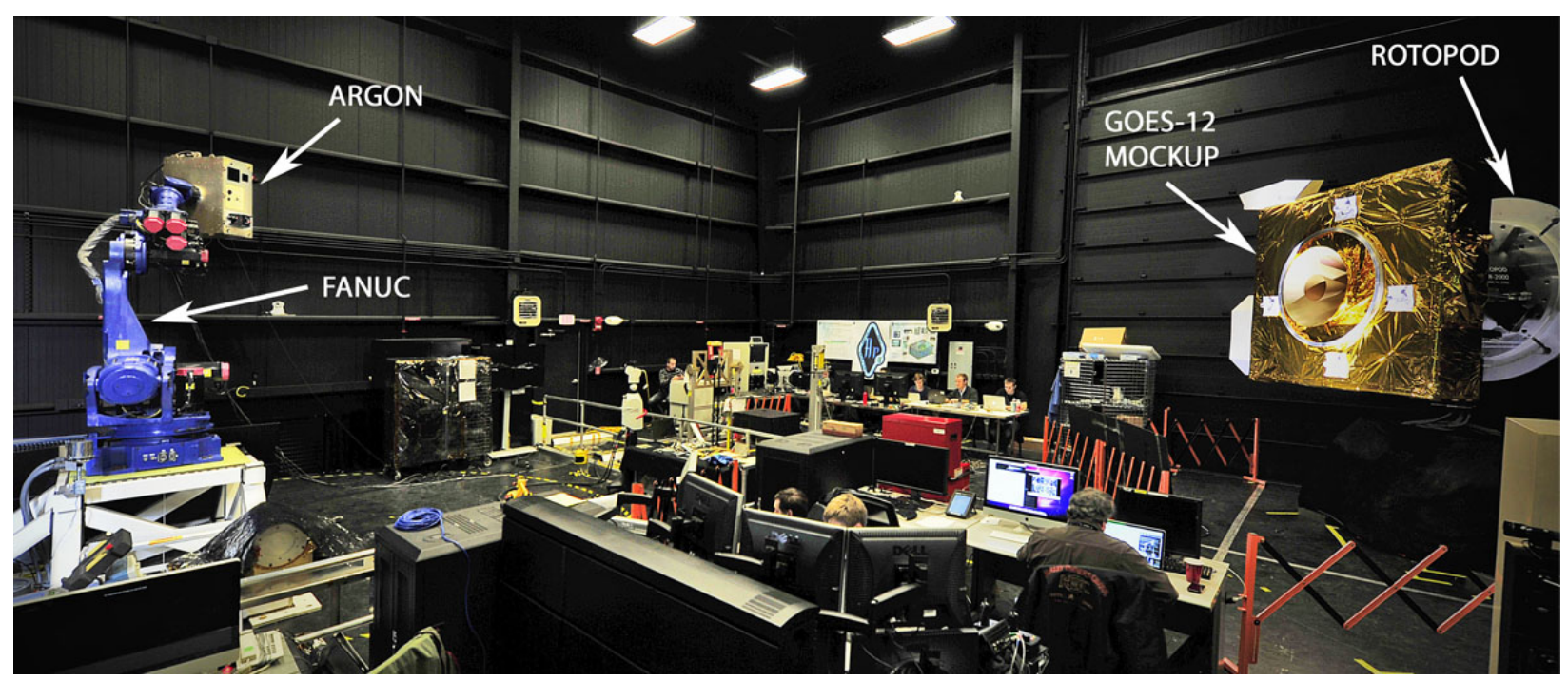

Figure 14. Argon test configuration at 10 meters separation in the SSCO Satellite Servicing Center. (Credit: NASA/Chris Gunn)

\section{Results}

The test plan had two independent sections: one evaluating pose performance evaluation, the focus of this discussion, and the other highlighting functional closed-loop testing. The pose evaluation runs consisted of relative motions that were isolated in each of the measured degrees of freedom, that is, during each test run, only one of the six relative degrees of freedom were actuated. In the translation directions, the commanded motion had a sawtooth velocity profile consisting of four cycles with period of 120 seconds; each cycle had a different peak velocity progressing through $1,3,5$, and $10 \mathrm{~cm} / \mathrm{sec}$. For the rotation directions, a similar profile was used except with shorter (60 seconds instead of 120) but more numerous cycles: starting at $0.01 \mathrm{deg} / \mathrm{sec}$ and stepping up to $0.1 \mathrm{deg} / \mathrm{sec}$ in $0.01 \mathrm{deg} / \mathrm{sec}$ increments; then 0.2 to $0.5 \mathrm{deg} / \mathrm{sec}$ in $0.1 \mathrm{deg} / \mathrm{sec}$ increments; and finally starting at $1.0 \mathrm{deg} / \mathrm{sec}$ to $5.0 \mathrm{deg} / \mathrm{sec}$ at $0.5 \mathrm{deg} / \mathrm{sec}$ intervals. Figure 15 displays GNFIR data for an example attitude test. The top two plots show translation and attitude error signals based on the induced target attitude rate that is shown in the bottom plot. For brevity, we only present results from the angular rate tests using the narrow FOV camera with a separation distance of 10 meters.

Pose solutions at each constant-rate plateau of the target attitude rate were reduced to a RMS mean and standard deviation value in order to display the overall position and attitude accuracy as a function of relative rates. Figure 16 shows position accuracy as a function of pitch and roll angular rates, while Figure 17 shows attitude accuracy for the same inputs. GNFIR's pose estimate errors responded similarly to target pitch and yaw rate, so only one set is presented here. While test data was gather for rates all the way up to $5.0 \mathrm{deg} / \mathrm{sec}$, to date only the roll rate tests up to $0.1 \mathrm{deg} / \mathrm{sec}$, pitch rate tests up to $0.5 \mathrm{deg} / \mathrm{sec}$, and yaw rate tests up to $0.5 \mathrm{deg} / \mathrm{sec}$ have been analyzed.

As expected, almost all GNFIR errors increase with increased target rates as the pose solution lags behind the moving target. This effect is directly related to the hardware under test, specifically the camera frame rate and CPU processing time. As the frame rate increases, the amount of pixels a feature traverses between 

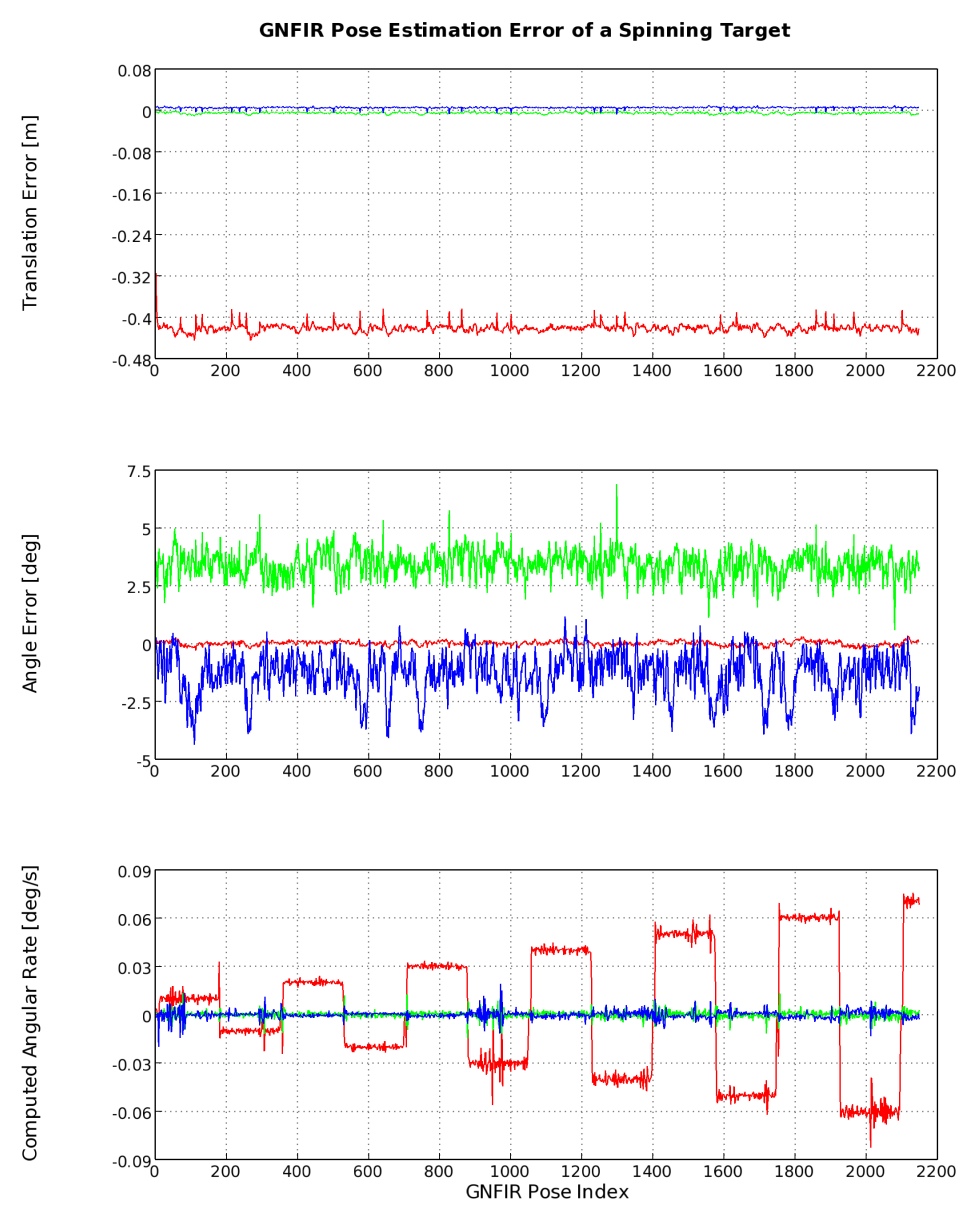

Figure 15. GNFIR data for attitude rate characterization test run with a ten meter separation distance. Blue, green, and red lines indicate translation, rotation, or rotation rate about the $\mathrm{x}, \mathrm{y}$, and $\mathrm{z}$ axes as appropriate (ie the red line on the Angle Error plot indicates yaw angle error).

frames goes down, allowing the algorithm to iterate on the same frame for fewer times in order to obtain a reasonable solution. Alternatively, one could increase the processing speed to allow more iterations to be completed on each frame which would effectively produce similar results to increasing the frame rate.

There is one GNFIR error that decreases with increasing target rates: the range estimate error decreases as target pitch rate increases. This same trend appears in the range estimate error for yaw rate tests which was omitted for brevity. While this may initially seem counterintuitive, a number of factors explain this result. First, pitch and yaw motion change the incident light angle for many features, unlike roll motion; features that are poorly lit may experience a favorable change in lighting. Second, transverse target rotation results in target feature movement in the range direction; this serves to excite the range axis of the GNFIR solution space. Third, the sensitivity of GNFIR to feature location model error in the model's transverse plane is reduced when the target undergoes a large transverse rotation; the model location error along the surface of the model is foreshortened due to the camera's perspective. Finally, large transverse rotations at the range that we performed these tests resulted in some of the target features to move out of the camera field of view; some of the features that dropped out of the camera field of view during this test were features that were the least well modeled.

\section{Relative Pose Tracking Performance Characterization}

The final set of tests were conducted at NRL's Proximity Operations Testbed (POT) facility during March and May of 2012. The primary objectives of this campaign were to demonstrate closed-loop control using live pose measurements and to evaluate the performance of the closed-loop system. One closed-loop test will 

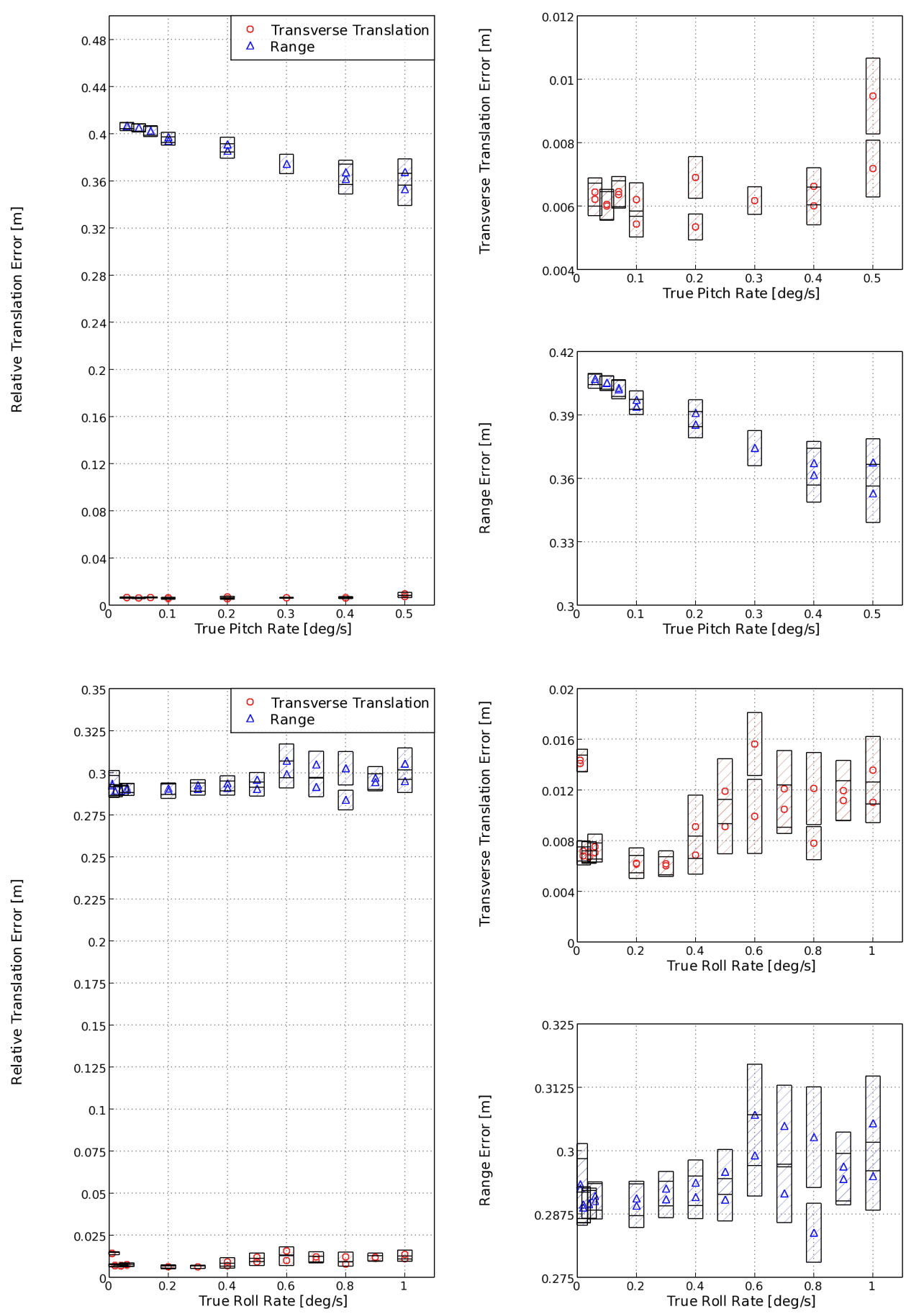

Figure 16. GNFIR position error as a function of in-plane attitude (top) and roll (bottom) rates. The left plots show the transverse and range errors on the same absolute scale. The right plots show transverse error independently of range errors and vice-versa in greater detail. 

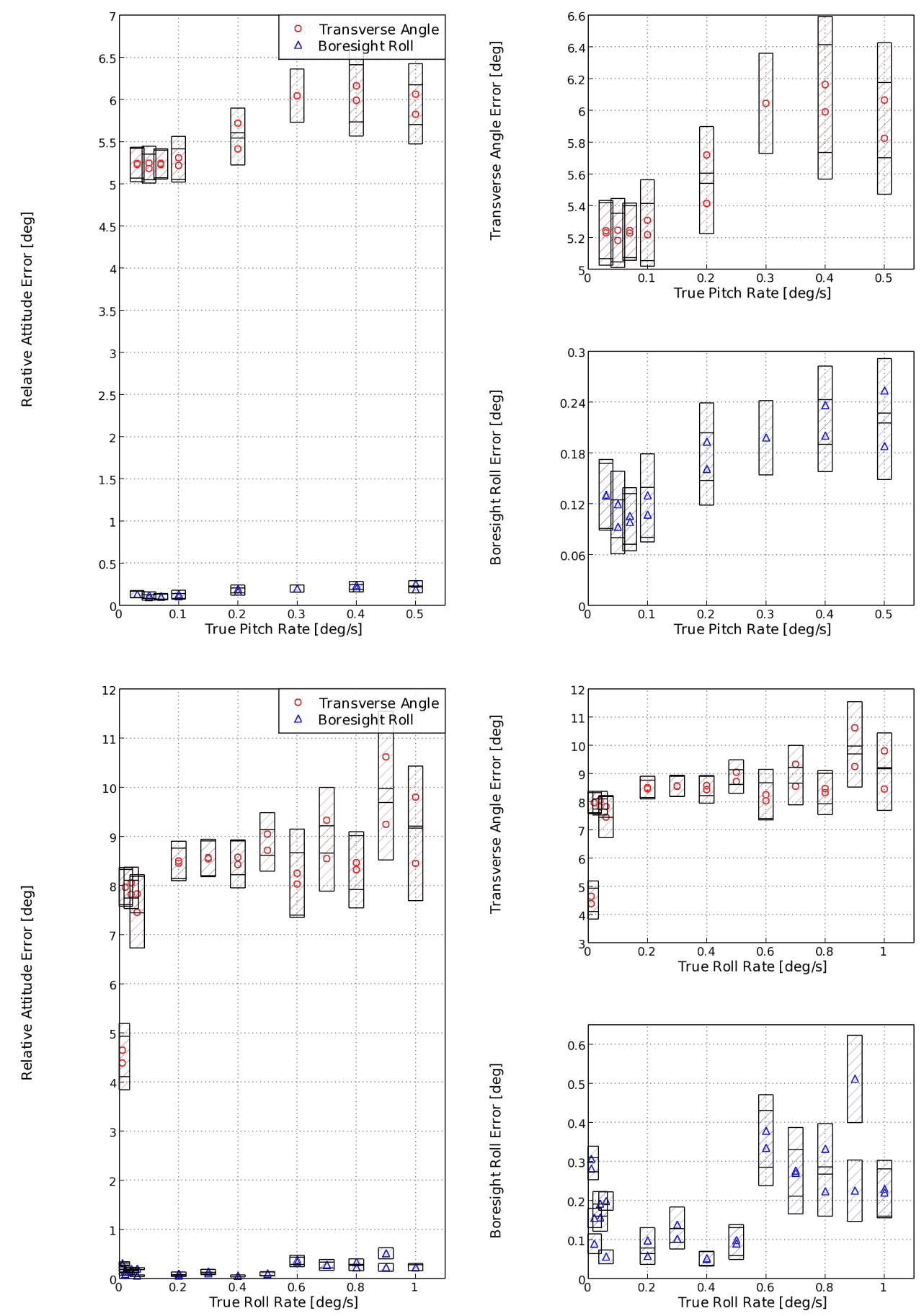

Figure 17. GNFIR attitude error as a function of in-plane attitude (top) and roll (bottom) rates. The left plots show the transverse and roll errors on the same absolute scale. The right plots show transverse error independently of roll errors and vice-versa in greater detail. 
be presented, wherein the Argon sensors and pose estimates were used to control a chaser (servicer) vehicle as it maneuvers through a series of hold points in the final phases of rendezvous with a static target (client).

\section{Experimental Setup}

Figure 18 shows the test setup at the POT facility. Two separate Cartesian gantries - one supporting a FANUC S900iw, the other a FANUC M900ia - are used to simulate the motion of a servicer and client spacecraft during the final phase of the rendezvous. The large workspace, roughly 9 meters wide by 2 meters tall by 20 meters long, allows the testing of nominal and off-nominal scenarios. For these tests, the lab frame was fixed to the orbit radial, in-track, and cross-track (RIC) frame, centered at the target center of mass; although, in general, any frame assignment is possible.

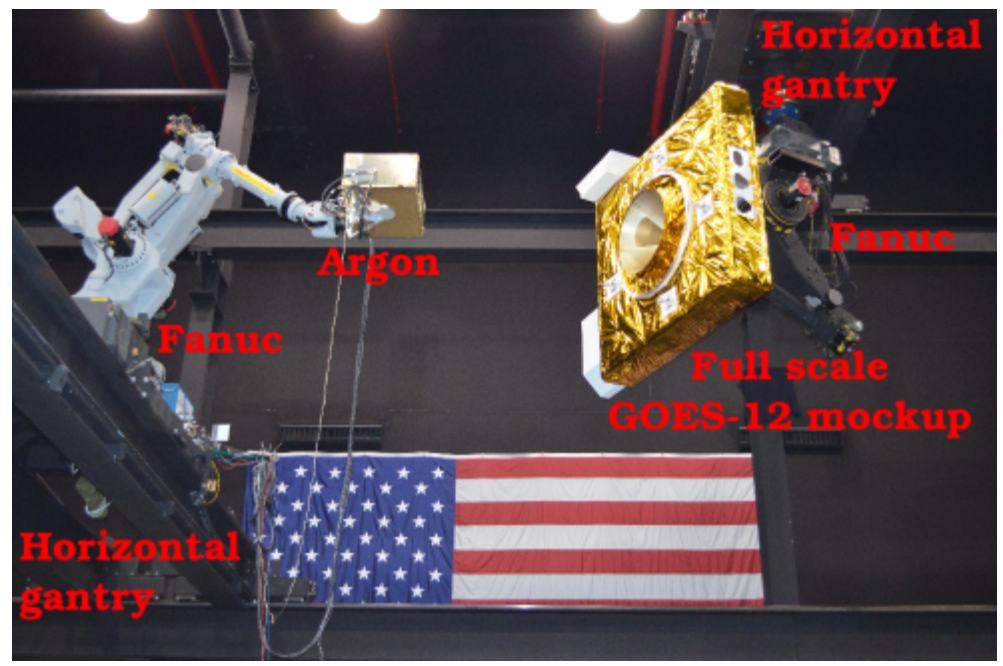

Figure 18. Argon test configuration at Naval Research Laboratory (NRL)'s Proximity Operations Testbed (POT) facility.

Figure 19 illustrates how Argon is used in the control loop. The pose measurements are relayed via ethernet to the simulation environment computer that implements filtering, guidance, and control laws; applies the forces and torques; and integrates the dynamics. Details of these algorithms can be found in Ref. 11. End effector locations with respect to the RIC frame are sent from the simulation computer to the NRL facility control computers which relay joint motion commands to the robots, which then move the "satellites" accordingly. Three separate GNFIR pose estimates are provided to the navigation filters at $2 \mathrm{~Hz}$. The filters compute a state update using the measurement only at $1 \mathrm{~Hz}$, but a high-rate propagator provides estimates to the controllers at $10 \mathrm{~Hz}$, which is the rate used in the dynamics simulation. The motion commands are computed and supplied to the facility at $5 \mathrm{~Hz}$. Though not shown on the figure, pose estimates from the Argon box were sent via ethernet to the Argon ground terminal and relayed to the Vispose graphical display tool.

Table 1 outlines the nominal, staged approach simulated in the lab. The sequence contains a multiple hold points that are representative of an actual orbital rendezvous. During individual test, the initial chaser vehicle location, the chaser target motion, the lighting conditions, and simulated sensor locations with respect to the chaser center of mass were all varied. The conditions chosen for the test results presented in this section accurately reflect the planned rendezvous with GOES-12. Of particular challenge was configuring a solar simulator (light source) with the appropriate incidence angles for the time of year in the simulation.

\section{Results}

Figure 20 shows the servicer trajectory during the autonomous rendezvous using only relative measurements from the Argon sensor suite. The plots on the left of the figure show the relative position and orientation of the servicer with respect to the RIC frame on the top and bottom, respectively. The plots on the right of Figure 20 show the corresponding velocities and rates. 


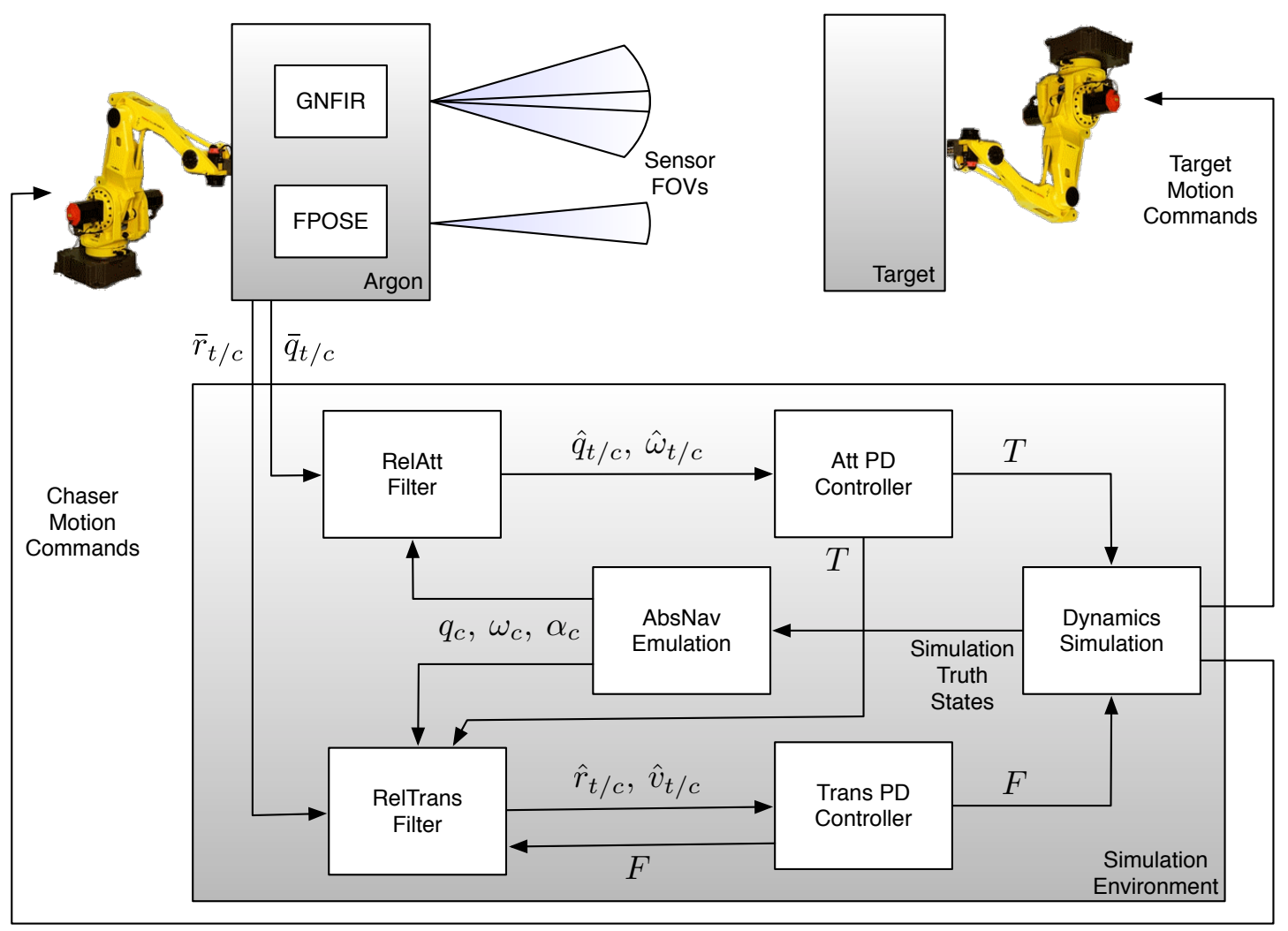

Figure 19. Control loop for servicer during final approach

Table 1. Timeline for the nominal approach sequence.

\begin{tabular}{ccccl} 
Stage & Type & $\begin{array}{c}\text { Duration/ } \\
\text { Speed }\end{array}$ & Separation & \multicolumn{1}{c}{ Description } \\
\hline 1 & Hold & $60 \mathrm{~s}$ & $17 \mathrm{~m}$ & $\begin{array}{l}\text { RIC stationkeeping, pitch/yaw at target, roll to } \\
\text { sun point solar arrays }\end{array}$ \\
\hline 2 & Move & $2.5 \mathrm{~cm} / \mathrm{s}$ & - & Approach target with same attitude pointing \\
\hline 3 & Hold & $300 \mathrm{~s}$ & $12 \mathrm{~m}$ & $\begin{array}{l}\text { RIC stationkeeping, align chaser and target body } \\
\text { axes }\end{array}$ \\
\hline 4 & Move & $2.5 \mathrm{~cm} / \mathrm{s}$ & - & Approach target with same attitude pointing \\
\hline 5 & Hold & $90 \mathrm{~s}$ & $9 \mathrm{~m}$ & $\begin{array}{l}\text { Stationkeep (relative translation and orientation) } \\
\text { for camera transition }\end{array}$ \\
\hline 6 & Move & $1.5 \mathrm{~cm} / \mathrm{s}$ & - & Approach to capture box \\
\hline 7 & Hold & $120 \mathrm{~s}$ & $2 \mathrm{~m}$ & Capture box stationkeeping
\end{tabular}



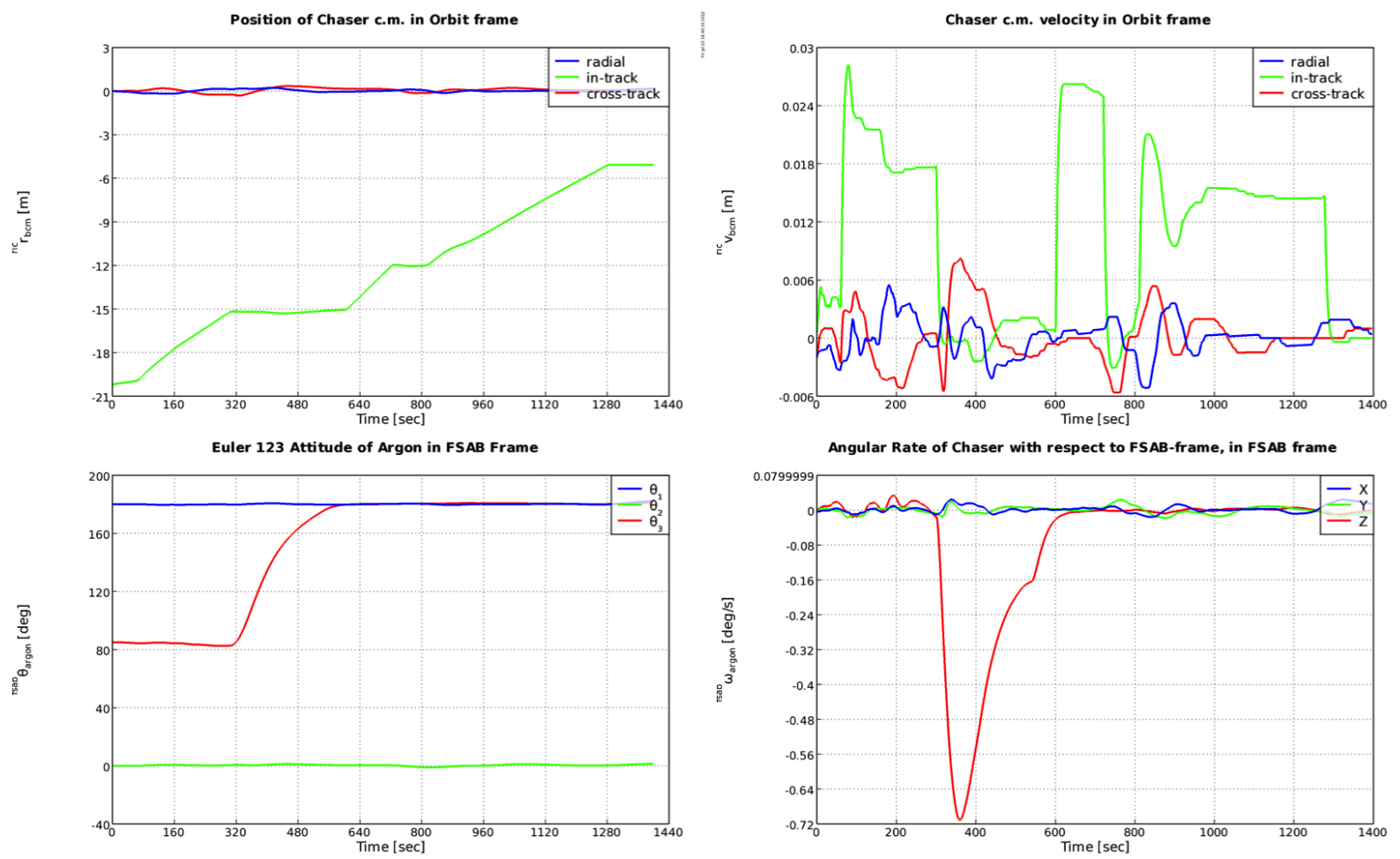

Figure 20. Closed-loop trajectory of servicer in orbit frame. Position and attitude on left and velocity and angular rates on right. Attitude is presented as 1-2-3 Euler angles.

The performance of the closed-loop system is heavily dependent on the accuracy of the pose measurement from GNFIR. Figure 21 shows how the accuracy and noise level is a strong function of range. The three instances of GNFIR running in Argon generated separate pose estimates. Two instances, GNFIR1 and GNFIR2, were configured to use the narrow Field of View (FOV) camera. The third instance, GNFIR3, used the wide FOV camera. This initial setup allowed for GNFIR1 and 2 to autonomously transition to the wide FOV camera when the solution using the other camera degraded below a set threshold (usually between 5 and 9 meters). Note that the error signal was set to zero for periods where GNFIR was in acquisition mode. The difference in performance is significant: GNFIR3 accuracy/noise is approximately a linear function of range, whereas GNFIR1 and GNFIR2 show more consistent performance throughout the test with the exception of the transition region where the target becomes too large in the FOV and key edges exit the image. This observation highlights the importance of maintaining a target-to-image size ratio within an optimal region. Estimation noise is significant when features cover too few pixels or when there are too few features in the image to resolve the pose estimate robustly.

To provide damping to the translational and attitude control loops, a Relative Navigation Filter (RNF) was developed to deliver estimates of velocity, angular rates, and noise-reduced estimates of pose to the satellite controller. Pose estimates from all three GNFIR instances, if in tracking mode, were processed by the filter and used to update the state estimate which was propagated at high rate for use in the controllers. The RNF consisted of a target inertial attitude filter and a relative translation filter. Perfect absolute measurements from a virtual ideal star tracker, Inertial Measurement Unit (IMU), and Global Positioning System Receiver (GPSR) were used to map the relative pose observations back into inertial space for propagation of target attitude (with the assumption of perfectly known inertias) and to form the orbital frame for RIC stationkeeping. The filter performance is shown in Figure 22. There was limited time to tune the filter, so the translation estimates did not perform to the level expected from simulations based on emulated measurements. After the initial convergence, the filter was unable to distinguish bias from state error, hence the state estimation error is greater than the mean sensor error. When the servicer performs a slew (320-580 s) to acquire capture attitude, this issue partially resolves and the error decreases. The lack of dynamic excitation in the latter part of the test, coupled with poorly modeled noise characteristics, lead to 

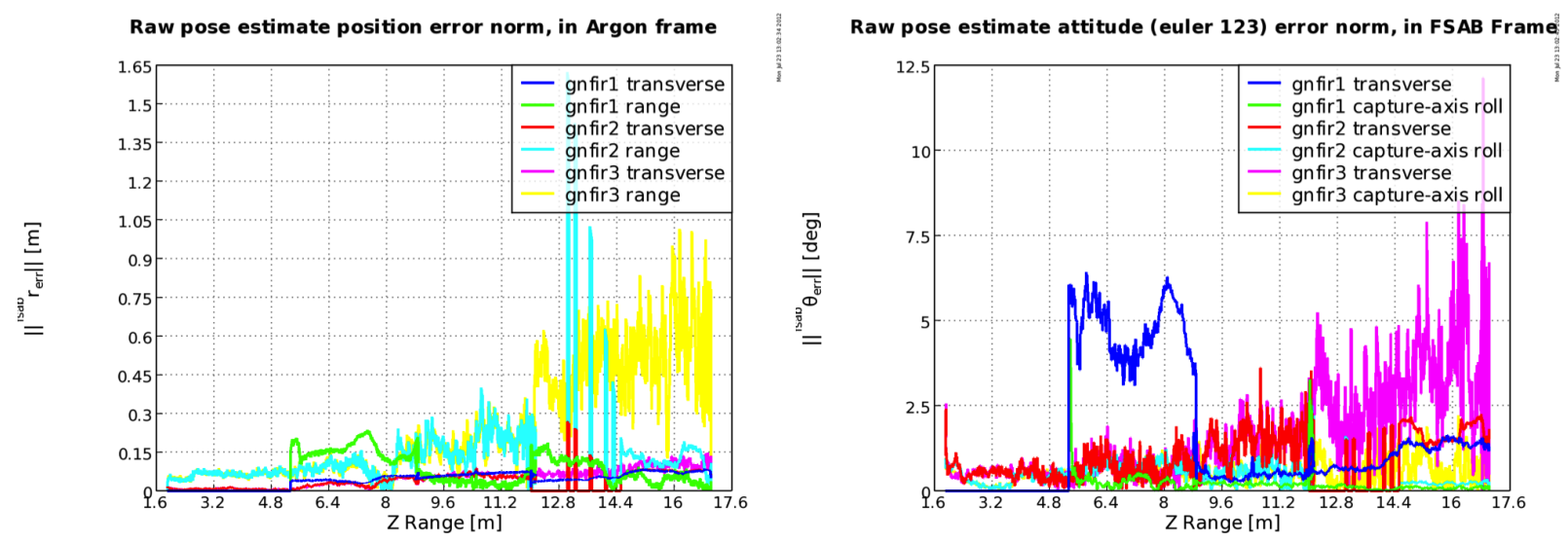

Figure 21. GNFIR accuracy as function of range for the nominal approach. The error signal is divided into the norm of the transverse $(x, y)$ and range $(z)$ components.

a repeat of this issue and significant drift of the estimated biases. The target attitude filter did not contain any bias states; as a result, its accuracy closely matched the mean accuracy of the GNFIR estimates with reduced noise levels. The rate estimates generally followed drift patterns in the pose estimate error signal but were sufficiently smooth for control.
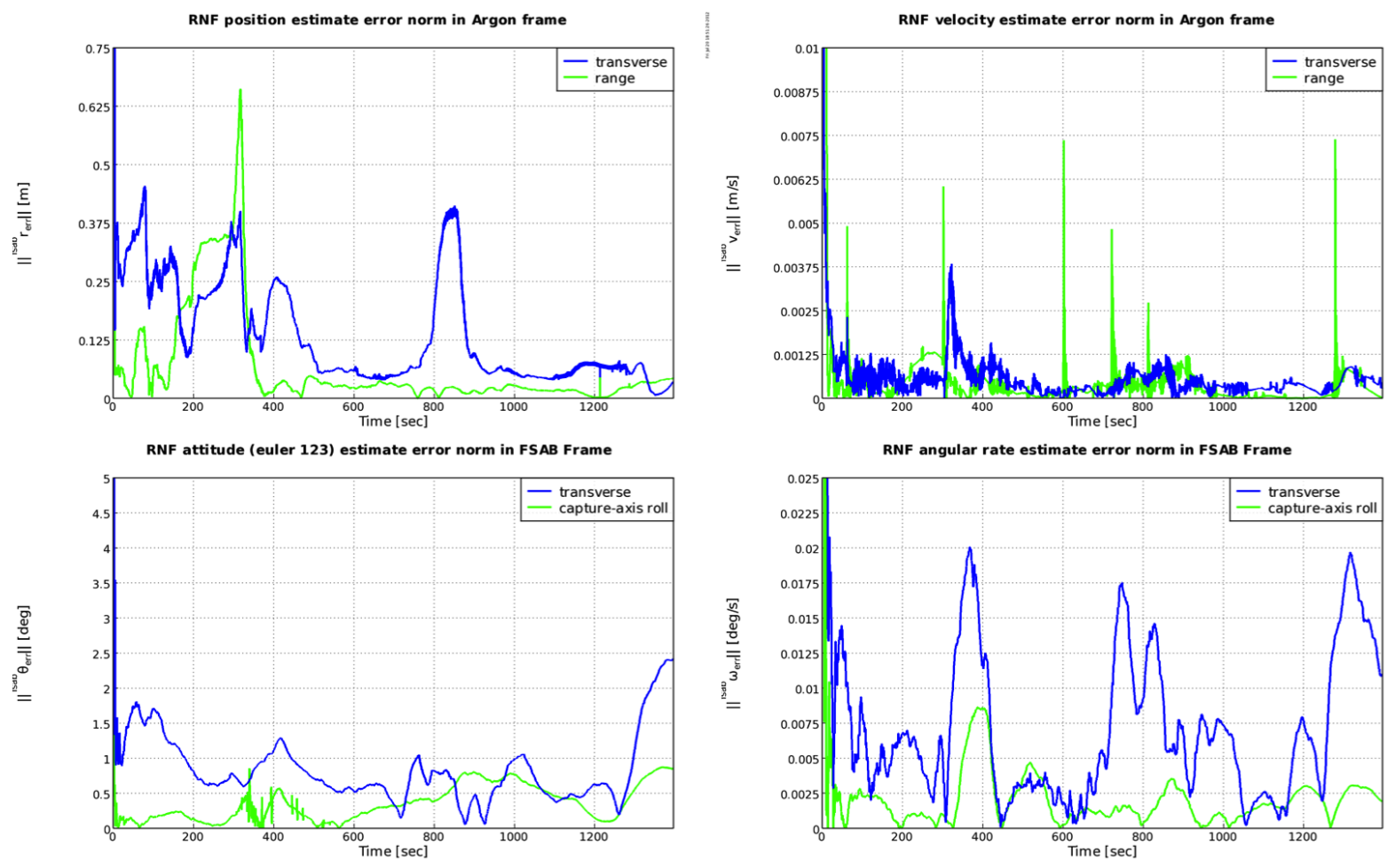

Figure 22. RNF pose and rate estimate accuracies. Position and attitude shown on the left and velocity and angular rates shown on the right.

The performance statistics for the nominal approach are quantified in Table 2. The different error types and their sources are described below.

- NRL facility pose error (NRL data vs. motion commands from dynamic simulator) is comprised of tracking latency/error in the gantries and FANUC robots. The most noticeable errors are the range 
bias $(\sim 3 \mathrm{~cm})$ and transient attitude error due to lag in tracking the servicer during its slew. The former arises from the low-accuracy of the laser sensors; the latter is due to the lack of a rate tracking (or feed forward term) in the FANUC pose controller.

- GNFIR pose error (pose measurement vs. NRL data) is more significant in the range direction (transverse bias is $\sim 2 \mathrm{~cm}$; range bias is $7-15 \mathrm{~cm}$ ), with greater bias present with the long range camera. Bias results from knowledge errors in the camera parameters and light-dependent differences between the observed/true edges and edges from the CAD model. Bias and noise were reasonably consistent between tests, including tests that were not published in this paper. However, the error statistics reported in the tables do not capture the range dependency as highlighted in Figure 21.

- RNF pose error (filter pose-state estimate vs. NRL data) contains significant walking bias, for reasons previously mentioned, but RNF rate error (filter rate estimate vs dynamic simulator data) is small.

- Test vs. sim. (dynamic simulation data from test vs. simulation using perfect measurements) evaluates the closed-loop performance of the system against a benchmark case obtained from a perfectmeasurement simulation, or the best possible trajectory given the controller and vehicle dynamics. In general the errors are of a similar magnitude to the filter errors.

- Final hold point tracking (NRL data during final hold vs. desired pose) is of particular interest to the GSFC robotics team, who, for a servicing mission, are responsible for designing a controller to grapple the target given the disturbance conditions or drift rates (if bus control is switched off) at the base of their manipulator. 


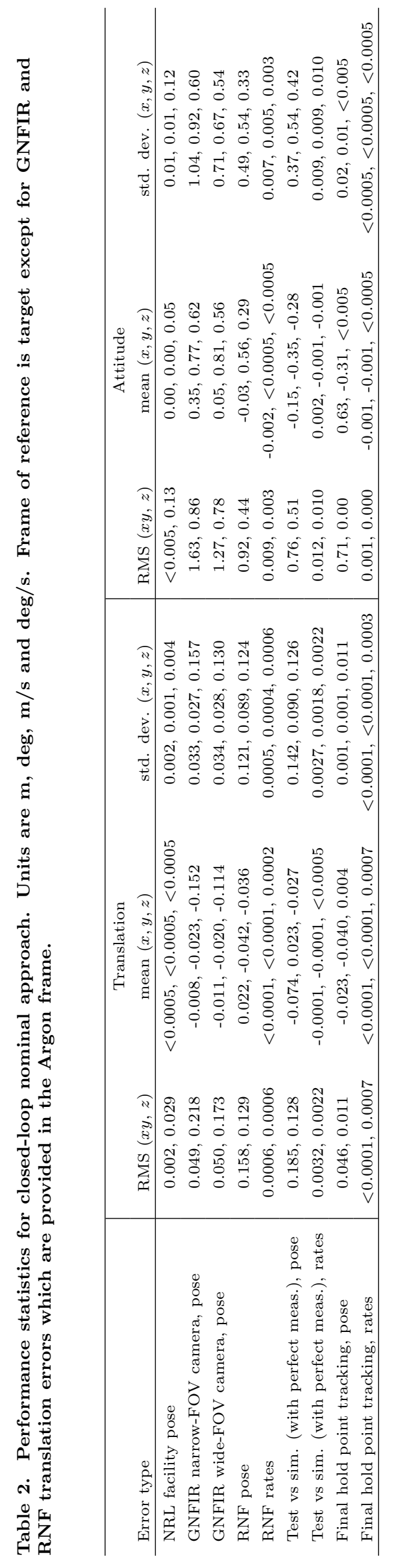

22 of 25

American Institute of Aeronautics and Astronautics 


\section{Conclusion}

Argon is a flight-ready sensor suite optimized for pose measurement in proximity, rendezvous, and docking operations. The Argon system performance was evaluated in a variety of locations against numerous variables such as separation distance, lighting conditions, and target rotation rates. GNFIR pose estimation error was constant over separation distances from 20 to 60 meters when using a 11 degree FOV camera, but showed increasing error with increased separation distances when using the wider 57 degree camera for distances from 17 to two meters. As expected, lighting had a significant impact in the performance of the algorithm. FPose displayed larger errors due to a pixel-to-pixel, range-and-setting-dependent biases in the LIDAR data.

GNFIR's sensitivity to target rotation rates was also examined. Most elements of GNFIR's pose estimate get worse as target rotation rate increases as expected; however, range estimates improve slightly as target transverse rotation rates increase as model sensitivity is reduced due to the apparent target foreshortening in the camera image and transverse rotation provides excitation in the range direction. For low enough rates, GNFIR's performance was comparable to performance when looking at a static target. This range spanned from 0 to $0.5 \mathrm{deg} / \mathrm{sec}$ for target roll rates and 0 to $0.1 \mathrm{deg} / \mathrm{sec}$ for target pitch or yaw rates. While GNFIR's performance degrades for higher rates (except in the case of range estimates during pitch/yaw motion as explained above), GNFIR was able to maintain good performance throughout the entire range of target rates analyzed as summarized in Table 3. Data for higher rates was collected but has yet to be analyzed. These specific performance results are of course dependent on the target geometry, model accuracy, lighting situation, relative separation distance, camera optics, and other factors.

\begin{tabular}{|r|cccc|}
\hline Target Motion & Range & Transverse Translation & Roll & Transverse Attitude \\
\hline \hline roll rate $\leq 0.5 \mathrm{deg} / \mathrm{sec}$ & 0.29 & 0.015 & 0.31 & 9.1 \\
roll rate $\leq 1 \mathrm{deg} / \mathrm{sec}$ & 0.31 & 0.016 & 0.51 & 10.6 \\
pitch/yaw rate $\leq 0.5 \mathrm{deg} / \mathrm{sec}$ & 0.41 & 0.007 & 0.14 & 5.3 \\
pitch/yaw rate $\leq 0.5 \mathrm{deg} / \mathrm{sec}$ & 0.41 & 0.010 & 0.25 & 6.2 \\
\hline
\end{tabular}

Table 3. Measured GNFIR Estimate Error Bounds During Rate Tests in meters or degrees as appropriate.

Finally, the Argon sensor suite was integrated in a closed-loop rendezvous and docking simulation system at a robotic test facility, where the Argon suite autonomously performed guidance, navigation, and control functionality for a simulated final approach to a representative satellite. When coupled with a RNF and a PD controller, the simulated robotic chase vehicle was capable of performing the following tasks relative to a simulated non-cooperative target satellite: stationkeep in a RIC frame, approach target while maintaining solar panel orientation to the Sun, align chaser to target and match rates, stationkeep in target frame, approach capture box, and stationkeep in target box.

\section{Acknowledgments}

The authors would like to thank the many people who made the Argon test campaign possible. We would like to thank the Argon test team of Ross Henry, Brian Tokarcik, Colin Vogel, Laura Walker, Jason Badgley, and Colin Eaton. We would also like to thank the flight software team of John Higinbotham, Will Clement, Terecita Mayorga, Chad Adebiyi, and Manuel Buenfil. For testing in the Satellite Servicing Center, we would like to thank Craig Carignan, Joseph Easley, Brian Gregory, and Alex Janas. Also, testing at POT facility couldn't have been done without help from NRL personnel, especially Glenn Creamer and Bernie Kelm. None of this would have been possible without support from the Satellite Servicing Capabilities Office (SSCO), Frank Cepollina, Ben Reed, and Bo Naasz.

\section{References}

\footnotetext{
${ }^{1}$ Ambrose, R., Wilcox, B., Reed, B., Matthies, L., Lavery, D., and Korsmeyer, D., "Space Technology Roadmap Technical Aera 04: Robotics, Tele-Robotics, and Autonomous Systems Roadmap," Tech. rep., NASA Office of the Chief Technologist, April 2012.

${ }^{2}$ Naasz, B. J., Strube, M., Eepoel, J. V., Barbee, B. W., and Getzandanner, K. M., "Satellite Servicings Autonomous Rendezvous and Docking Testbed on the International Space Station," Proceedings of the 34th AAS Guidance and Control Conference, No. AAS 11-072, Breckenridge Colorado.
} 
${ }^{3}$ Naasz, B. J., Eepoel, J. V., Queen, S. Z., II, C. M. S., and Hannah, J., "Flight Results From the HST SM4 Relative Navigation Sensor System," Proceedings of the 33rd AAS Guidance and Control Conference, No. AAS 10-086, Breckenridge Colorado.

${ }^{4}$ Christian, J. A., Hinkel, H., D’Souza, C. N., Maguire, S., and Patangan, M., "The Sensor Test for Orion RelNav Risk Mitigation (STORRM) Development Test Objective," Proceedings of the 2011 AIAA Guidance, Navigation, and Control Conference, No. AAS 11-6260.

${ }^{5}$ Drummond, T. and Cipolla, R., "Real-Time Visual Tracking of Complex Structures," IEEE Transactions on Pattern Analysis and Machine Intelligence, 2002.

${ }^{6}$ Besl, P. J. and McKay, N. D., "A Method for Registration of 3-D Shapes," IEEE Transactions of Pattern Analysis and Machine Intelligence, Vol. 14, No. 2, February 1992, pp. 239-256.

${ }^{7}$ Center, N. G. S. F., "SpaceCube to Debut in Flight Experiment: Hybrid Computer to Fly on Hubble Servicing Mission," http://gsfctechnology.gsfc.nasa.gov/SpaceCube.htm, July 2012.

8 "Advanced Spacecraft Integration \& System Test Software (ASIST)," http://nasa-asist.gsfc.nasa.gov/, July 2012, NASA Goddard Space Flight Center.

${ }^{9}$ Christian, J. A., Patangan, M., Hinkel, H., and Chevray, K., "Comparison of Orion Vision Navigation Sensor Performance from STS-134 and the Space Operations Simulation Center," AIAA Guidance, Navigation, and Control Conference 2012, Minneapolis, MN.

${ }^{10}$ Center, N. G. S. F., "Robotic Refueling Mission," Jun 2011, FS-2011-3-112-GSFC.

${ }^{11}$ Carignan, C. R., Easley, J. W., Hyslop, A. M., and Strube, M. J., "Ground Simulation of an Autonomous Satellite Rendezvous and Tracking System Using Dual Robotic Systems," Proceedings of the 2012 International Symposium on Artificial Intelligence, Robotics and Automation in Space Conference, Turin, Italy.

\section{Acronyms and Abbreviations}

AGC automatic gain control

ASIST Advanced Spacecraft Integration \& System Test Software

DPP Dextre Pointing Package

DTO Demonstration Test Objective

EDU engineering development unit

FOV Field of View

FPGA Field Programmable Gate Array

FPose FlashPose

GNFIR Goddard Natural Feature Image Recognition

GPSR Global Positioning System Receiver

GSFC Goddard Space Flight Center

HST Hubble Space Telescope

ICP Iterative Closest Point

IMU Inertial Measurement Unit

ISS International Space Station

LIDAR Light Detection and Ranging

MDA MacDonald, Dettwiler and Associates

MPCV Multi-Purpose Crew Vehicle

NESC NASA Engineering and Safety Center

NRL Naval Research Laboratory

POT Proximity Operations Testbed 
RMS Root Mean Square

RNF Relative Navigation Filter

RNS Relative Navigation Sensor

RPO Rendezvous and Proximity Operations

RRM Robotic Refueling Mission

SM4 Servicing Mission 4

SSCO Satellite Servicing Capabilities Office

STORRM Sensor Test for Orion Rel-Nav Risk Mitigation

VCM Video Control Module

VNS Vision Navigation System 
26 of 25 